\title{
Massless bound-state excitations and the Schwinger mechanism in QCD
}

\author{
A.C. Aguilar, ${ }^{1}$ D. Ibáñez, ${ }^{2}$ V. Mathieu, ${ }^{2}$ and J. Papavassiliou ${ }^{2}$ \\ ${ }^{1}$ Federal University of $A B C, C C N H$, \\ Rua Santa Adélia 166, CEP 09210-170, Santo André, Brazil. \\ ${ }^{2}$ Department of Theoretical Physics and IFIC, \\ University of Valencia-CSIC, E-46100, Valencia, Spain.
}

\begin{abstract}
The gauge invariant generation of an effective gluon mass proceeds through the well-known Schwinger mechanism, whose key dynamical ingredient is the nonperturbative formation of longitudinally coupled massless bound-state excitations. These excitations introduce poles in the vertices of the theory, in such a way as to maintain the Slavnov-Taylor identities intact in the presence of massive gluon propagators. In the present work we first focus on the modifications induced to the nonperturbative three-gluon vertex by the inclusion of massless two-gluon bound-states into the kernels appearing in its skeleton-expansion. Certain general relations between the basic building blocks of these bound-states and the gluon mass are then obtained from the Slavnov-Taylor identities and the Schwinger-Dyson equation governing the gluon propagator. The homogeneous Bethe-Salpeter equation determining the wave-function of the aforementioned bound state is then derived, under certain simplifying assumptions. It is then shown, through a detailed analytical and numerical study, that this equation admits non-trivial solutions, indicating that the QCD dynamics support indeed the formation of such massless bound states. These solutions are subsequently used, in conjunction with the aforementioned relations, to determine the momentum-dependence of the dynamical gluon mass. Finally, further possibilities and open questions are briefly discussed.
\end{abstract}

PACS numbers: $12.38 . \mathrm{Lg}, 12.38 . \mathrm{Aw}, 12.38 . \mathrm{Gc}$ 


\section{INTRODUCTION}

The numerous large-volume lattice simulations carried out in recent years have firmly established that, in the Landau gauge, the gluon propagator and the ghost dressing function of pure Yang-Mills theories are infrared finite, both in $S U(2)$ [1 $[5]$ and in $S U(3)$ [6 6 ] . Perhaps the most physical way of explaining the observed finiteness of these quantities is the generation of a non-perturbative, momentum-dependent gluon mass [10 15], which acts as a natural infrared cutoff. In this picture the fundamental Lagrangian of the Yang-Mills theory (or that of QCD) remains unaltered, and the generation of the gluon mass takes place dynamically, through the well-known Schwinger mechanism [16-22] without violating any of the underlying symmetries (for further studies and alternative approaches, see, e.g., [23-29] ).

The way how the Schwinger mechanism generates a mass for the gauge boson (gluon) can be seen most directly at the level of its inverse propagator, $\Delta^{-1}\left(q^{2}\right)=q^{2}\left[1+i \Pi\left(q^{2}\right)\right]$, where $\boldsymbol{\Pi}(q)$ is the dimensionless vacuum polarization. According to Schwinger's fundamental observation, if $\Pi\left(q^{2}\right)$ develops a pole at zero momentum transfer $\left(q^{2}=0\right)$, then the vector meson acquires a mass, even if the gauge symmetry forbids a mass term at the level of the fundamental Lagrangian. Indeed, if $\Pi\left(q^{2}\right)=m^{2} / q^{2}$, then (in Euclidean space) $\Delta^{-1}\left(q^{2}\right)=q^{2}+m^{2}$, and so the vector meson becomes massive, $\Delta^{-1}(0)=m^{2}$, even though it is massless in the absence of interactions $(g=0, \Pi=0)$ [18, 19].

The key assumption when invoking the Schwinger mechanism in Yang-Mills theories, such as QCD, is that the required poles may be produced due to purely dynamical reasons; specifically, one assumes that, for sufficiently strong binding, the mass of the appropriate bound state may be reduced to zero [18 22]. In addition to triggering the Schwinger mechanism, these massless composite excitations are crucial for preserving gauge invariance. Specifically, the presence of massless poles in the off-shell interaction vertices guarantees that the Ward identities (WIs) and Slavnov Taylor identities (STIs) of the theory maintain exactly the same form before and after mass generation (i.e. when the the massless propagators appearing in

them are replaced by massive ones) [10, 15, 21, 22]. Thus, these excitations act like dynamical Nambu-Goldstone scalars, displaying, in fact, all their typical characteristics, such as masslessness, compositeness, and longitudinal coupling; note, however, that they differ from Nambu-Goldstone bosons as far as their origin is concerned, since they are not associated 
with the spontaneous breaking of any global symmetry [10]. Finally, every such Goldstonelike scalar, "absorbed" by a gluon in order to acquire a mass, is expected to actually cancel out of the $S$-matrix against other massless poles or due to current conservation [18-22].

The main purpose of the present article is to scrutinize the central assumption of the dynamical scenario outlined above, namely the possibility of actual formation of such massless excitations. The question we want to address is whether the non-perturbative Yang Mills dynamics are indeed compatible with the generation of such a special bound-state. In particular, as has already been explained in previous works, the entire mechanism of gluon mass generation hinges on the appearance of massless poles inside the nonperturbative three-gluon vertex, which enters in the Schwinger Dyson equation (SDE) governing the gluon propagator. These poles correspond to the propagator of the scalar massless excitation, and interact with a pair of gluons through a very characteristic proper vertex, which, of course, must be non vanishing, or else the entire construction collapses. The way to establish the existence of this latter vertex is through the study of the homogeneous Bethe-Salpeter equation (BSE) that it satisfies, and look for non-trivial solutions, subject to the numerous stringent constraints imposed by gauge invariance.

This particular methodology has been adopted in various early contributions on this subject; however, only asymptotic solutions to the corresponding equations have been considered. The detailed numerical study presented here demonstrates that, under certain simplifying assumptions for the structure of its kernel, the aforementioned integral equation has indeed non-trivial solutions, valid for the entire range of physical momenta. This result, although approximate and not fully conclusive, furnishes additional support in favor of the concrete mass generation mechanism described earlier.

The article is organized as follows. In Section [I] we set up the general theoretical framework related to the gauge-invariant generation of a gluon mass; in particular, we outline how the vertices of the theory must be modified, through the inclusion of longitudinally coupled massless poles, in order to maintain the WIs and STIs of the theory intact. In Section [II] we take a detailed look into the structure of the non-perturbative vertex that contains the required massless poles, and study its main dynamical building blocks, and in particular the transition amplitude between a gluon and a massless excitation and the proper vertex function (bound-state wave function), controlling the interaction of the massless excitation with two gluons. In addition, we derive an exact relation between these two quantities and 
the first derivative of the (momentum-dependent) gluon mass. Then, we derive a simple formula that, at zero momentum transfer, relates the aforementioned transition amplitude to the gluon mass. In the next two sections we turn to the central question of this work, namely the dynamical realization of the massless excitation within the Yang-Mills theory. Specifically, in Section [V] we derive the BSE that the proper vertex function satisfies, and implement a number of simplifying assumptions. Then, in Section $\nabla$ we demonstrate through a detailed numerical study that the resulting homogeneous integral equation admits indeed non-trivial solutions, thus corroborating the existence of the required bound-state excitations. In Section VI we demonstrate with a specific example the general mechanism that leads to the decoupling of all massless poles from the physical (on-shell) amplitude. Finally, in Section VII we discuss our results and present our conclusions.

\section{GENERAL CONSIDERATIONS}

In this section, after establishing the necessary notation, we briefly review why the dynamical generation of a mass is inextricably connected to the presence of a special vertex, which exactly compensates for the appearance of massive instead of massless propagators in the corresponding WIs and STIs.

The full gluon propagator $\Delta_{\mu \nu}^{a b}(q)=\delta^{a b} \Delta_{\mu \nu}(q)$ in the Landau gauge is defined as

$$
\Delta_{\mu \nu}(q)=-i P_{\mu \nu}(q) \Delta\left(q^{2}\right)
$$

where

$$
P_{\mu \nu}(q)=g_{\mu \nu}-\frac{q_{\mu} q_{\nu}}{q^{2}}
$$

is the usual transverse projector, and the scalar cofactor $\Delta\left(q^{2}\right)$ is related to the (all-order) gluon self-energy $\Pi_{\mu \nu}(q)=P_{\mu \nu}(q) \Pi\left(q^{2}\right)$ through

$$
\Delta^{-1}\left(q^{2}\right)=q^{2}+i \Pi\left(q^{2}\right)
$$

One may define the dimensionless vacuum polarization $\Pi\left(q^{2}\right)$ by setting $\Pi\left(q^{2}\right)=q^{2} \Pi\left(q^{2}\right)$ so that $(\underline{2.3})$ becomes

$$
\Delta^{-1}\left(q^{2}\right)=q^{2}\left[1+i \boldsymbol{\Pi}\left(q^{2}\right)\right]
$$

As explained in the Introduction, if $\Pi\left(q^{2}\right)$ develops at zero momentum transfer a pole with positive residue $m^{2}$, then $\Delta^{-1}(0)=m^{2}$, and the gluon is endowed with an effective mass. 


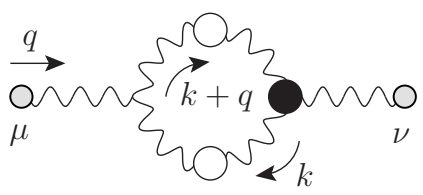

$\left(a_{1}\right)$

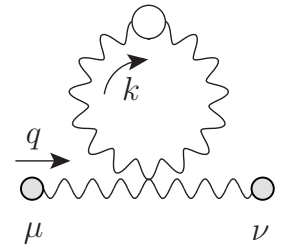

$\left(a_{2}\right)$

FIG. 1: The "one-loop dressed" gluon contribution to the PT-BFM gluon self-energy. White (black) circles denote fully dressed propagators (vertices); a gray circle attached to the external legs indicates that they are background gluons. Within the PT-BFM framework these two diagrams constitute a transverse subset of the full gluon SDE.

Alternatively, one may define the gluon dressing function $J\left(q^{2}\right)$ as

$$
\Delta^{-1}\left(q^{2}\right)=q^{2} J\left(q^{2}\right)
$$

In the presence of a dynamically generated mass, the natural form of $\Delta^{-1}\left(q^{2}\right)$ is given by (Euclidean space)

$$
\Delta^{-1}\left(q^{2}\right)=q^{2} J\left(q^{2}\right)+m^{2}\left(q^{2}\right)
$$

where the first term corresponds to the "kinetic term", or "wave function" contribution, whereas the second is the (positive-definite) momentum-dependent mass. If one insist on maintaining the form of (2.5) by explicitly factoring out a $q^{2}$, then

$$
\Delta^{-1}\left(q^{2}\right)=q^{2}\left[J\left(q^{2}\right)+\frac{m^{2}\left(q^{2}\right)}{q^{2}}\right],
$$

and the presence of the pole, with residue given by $m^{2}(0)$, becomes manifest.

Of course, in order to obtain the full dynamics, such as, for example, the momentumdependence of the dynamical mass, one must turn eventually to the SDE that governs the corresponding gauge-boson self-energy (see Fig. 1). In what follows we will work within the specific framework provided by the synthesis of the pinch technique (PT) [10, 30 34] with the background field method (BFM) [35]. One of the main advantages of the "PT-BFM" formalism is that the crucial transversality property of the gluon self-energy $\Pi_{\mu \nu}(q)$, namely $q^{\mu} \Pi_{\mu \nu}(q)=0$, is maintained at the level of the truncated SDEs [12, 36].

The Schwinger mechanism is integrated into the SDE of the gluon propagator through the form of the three-gluon vertex. In particular, as has been emphasized in some of the 
literature cited above (e.g.,[15]), a crucial condition for the realization of the gluon mass generation scenario is the existence of a special vertex, to be denoted by $V_{\alpha \mu \nu}(q, r, p)$ which must be completely longitudinally coupled, i.e. must satisfy

$$
P^{\alpha^{\prime} \alpha}(q) P^{\mu^{\prime} \mu}(r) P^{\nu^{\prime} \nu}(p) V_{\alpha \mu \nu}(q, r, p)=0
$$

We will refer to this special vertex as the "pole vertex" or simply "the vertex $V$ ".

The role of the vertex $V_{\alpha \mu \nu}(q, r, p)$ is indispensable for maintaining gauge invariance, given that the massless poles that it must contain in order to trigger the Schwinger mechanism, act, at the same time, as composite, longitudinally coupled Nambu-Goldstone bosons. Specifically, in order to preserve the gauge-invariance of the theory in the presence of masses, the vertex $V_{\alpha \mu \nu}(q, r, p)$ must be added to the conventional (fully-dressed) three-gluon vertex $\mathbb{\Gamma}_{\alpha \mu \nu}(q, r, p)$, giving rise to the new full vertex, $\mathbb{\Gamma}_{\alpha \mu \nu}^{\prime}(q, r, p)$, defined as

$$
\mathbb{\Gamma}_{\alpha \mu \nu}^{\prime}(q, r, p)=\mathbb{\Gamma}_{\alpha \mu \nu}(q, r, p)+V_{\alpha \mu \nu}(q, r, p)
$$

Gauge-invariance remains intact because $\mathbb{\Gamma}^{\prime}$ satisfies the same STIs as $\mathbb{\Gamma}$ before, but now replacing the gluon propagators appearing on their rhs by a massive ones; schematically, $\Delta^{-1} \rightarrow \Delta_{m}^{-1}$, where the former denotes the propagator given in (2.5), while the latter that of (2.6). In particular, in the PT-BFM framework that we employ, the vertex $\mathbb{\Gamma}$ connects a background gluon $(B)$ with two quantum gluons $(Q)$, and is often referred to as the "BQQ" vertex. This vertex satisfies a (ghost-free) WI when contracted with the momentum $q_{\alpha}$ of the background gluon, whereas it satisfies a STI when contracted with the momentum $r_{\mu}$ or $p_{\nu}$ of the quantum gluons. In particular,

$$
\begin{aligned}
& q^{\alpha} \mathbb{\Gamma}_{\alpha \mu \nu}(q, r, p)=p^{2} J\left(p^{2}\right) P_{\mu \nu}(p)-r^{2} J\left(r^{2}\right) P_{\mu \nu}(r), \\
& r^{\mu} \mathbb{\Gamma}_{\alpha \mu \nu}(q, r, p)=F\left(r^{2}\right)\left[q^{2} \widetilde{J}\left(q^{2}\right) P_{\alpha}^{\mu}(q) H_{\mu \nu}(q, r, p)-p^{2} J\left(p^{2}\right) P_{\nu}^{\mu}(p) \widetilde{H}_{\mu \alpha}(p, r, q)\right], \\
& p^{\nu} \mathbb{\Gamma}_{\alpha \mu \nu}(q, r, p)=F\left(p^{2}\right)\left[r^{2} J\left(r^{2}\right) P_{\mu}^{\nu}(r) \widetilde{H}_{\nu \alpha}(r, p, q)-q^{2} \widetilde{J}\left(q^{2}\right) P_{\alpha}^{\nu}(q) H_{\nu \mu}(q, p, r)\right],
\end{aligned}
$$

where $F\left(q^{2}\right)$ is the "ghost dressing function", defined as $F\left(q^{2}\right)=q^{2} D\left(q^{2}\right), H_{\nu \sigma}$ is the standard gluon-ghost kernel, and $\widetilde{H}$ is the same as $H$ but with the external quantum gluon replaced by a background gluon. Similarly, $\widetilde{J}$ is the dressing function of the self-energy connecting a background with a quantum gluon; $\widetilde{J}$ is related to $J\left(q^{2}\right)$ through the identity [37, 38]

$$
\widetilde{J}\left(q^{2}\right)=\left[1+G\left(q^{2}\right)\right] J\left(q^{2}\right) .
$$


The function $G\left(q^{2}\right)$ is the scalar co-factor of the $g_{\mu \nu}$ component of the special two-point function $\Lambda_{\mu \nu}(q)$, defined as

$$
\begin{aligned}
\Lambda_{\mu \nu}(q) & =-i g^{2} C_{A} \int_{k} \Delta_{\mu}^{\sigma}(k) D(q-k) H_{\nu \sigma}(-q, q-k, k) \\
& =g_{\mu \nu} G\left(q^{2}\right)+\frac{q_{\mu} q_{\nu}}{q^{2}} L\left(q^{2}\right) .
\end{aligned}
$$

Note finally that, in the Landau gauge, $G\left(q^{2}\right)$ and $L\left(q^{2}\right)$ are linked to $F\left(q^{2}\right)$ by the exact (all-order) relation [39-42]

$$
F^{-1}\left(q^{2}\right)=1+G\left(q^{2}\right)+L\left(q^{2}\right),
$$

to be employed in Subsection D.

Returning to the nonperturbative vertex $V$, gauge invariance requires that it must satisfy the WI and STI of (2.10), with the replacement $k^{2} J(k) \rightarrow-m^{2}(k)$, e.g.,

$$
q^{\alpha} V_{\alpha \mu \nu}(q, r, p)=m^{2}\left(r^{2}\right) P_{\mu \nu}(r)-m^{2}\left(p^{2}\right) P_{\mu \nu}(p) ;
$$

exactly analogous expressions will hold for the STIs satisfied when contracting with the momenta $r$ or $p$. Indeed, under this assumption, the full vertex $\mathbb{\Gamma}^{\prime}$ will satisfy the same WI and STIs as the vertex $\mathbb{\Gamma}$ before the introduction of any masses, but now with the replacement $q^{2} J\left(q^{2}\right) \rightarrow q^{2} J\left(q^{2}\right)+m^{2}\left(q^{2}\right)$. Specifically, combining the first relation in (2.10) with (2.14), one obtains for the WI of $\mathbb{\Gamma}^{\prime}$,

$$
\begin{aligned}
q^{\alpha} \Gamma_{\alpha \mu \nu}^{\prime}(q, r, p) & =q^{\alpha}[\mathbb{\Gamma}(q, r, p)+V(q, r, p)]_{\alpha \mu \nu} \\
& =\left[p^{2} J\left(p^{2}\right)-m^{2}\left(p^{2}\right)\right] P_{\mu \nu}(p)-\left[r^{2} J\left(r^{2}\right)-m^{2}\left(r^{2}\right)\right] P_{\mu \nu}(r) \\
& =\Delta_{m}^{-1}\left(p^{2}\right) P_{\mu \nu}(p)-\Delta_{m}^{-1}\left(r^{2}\right) P_{\mu \nu}(r),
\end{aligned}
$$

which is indeed the first identity in Eq. (2.10), with the aforementioned replacement $\Delta^{-1} \rightarrow \Delta_{m}^{-1}$ enforced. The remaining two STIs are realized in exactly the same fashion.

It must be clear at this point that the longitudinal nature of $V_{\alpha \mu \nu}$, combined with the WI and STIs that it must satisfy, lead inevitably to the appearance of a massless pole, as required by the Schwinger mechanism. For example, focusing only on the $q$-channel, the simplest toy Ansatz for the vertex is

$$
V_{\alpha \mu \nu}(q, r, p)=\frac{q_{\mu}}{q^{2}}\left[m^{2}\left(r^{2}\right) P_{\mu \nu}(r)-m^{2}\left(p^{2}\right) P_{\mu \nu}(p)\right],
$$

which has a pole in $q^{2}$ and satisfies (2.14). Of course, poles associated to the other channels $\left(r\right.$ and $p$ ) will also appear, given that $V_{\alpha \mu \nu}(q, r, p)$ must also satisfy the corresponding STIs with respect to $r^{\mu}$ and $p^{\nu}$. 


\section{THE POLE VERTEX: STRUCTURE AND PROPERTIES}

In this section we have a detailed look at the structure of the special vertex $V$. In particular, we identify the diagrammatic origin and field-theoretic nature of the various quantities contributing to it, and specify the way it enters into the SDE of the full vertex $\mathbb{I}^{\prime}$, defined in Eq. (2.9). In addition, we will derive an exact relation between the most important component of this vertex and the derivative of the momentum-dependent gluon mass.

\section{A. General structure of the vertex $V$}

The main characteristic of the vertex $V$, which sharply differentiates it from ordinary vertex contributions, is that it contains massless poles, originating from the contributions of bound-state excitations. Specifically, all terms of the vertex $V$ are proportional to $1 / q^{2}, 1 / r^{2}$, $1 / p^{2}$, and products thereof. Such dynamically generated poles are to be clearly distinguished from poles related to ordinary massless propagators, associated with elementary fields in the original Lagrangian.

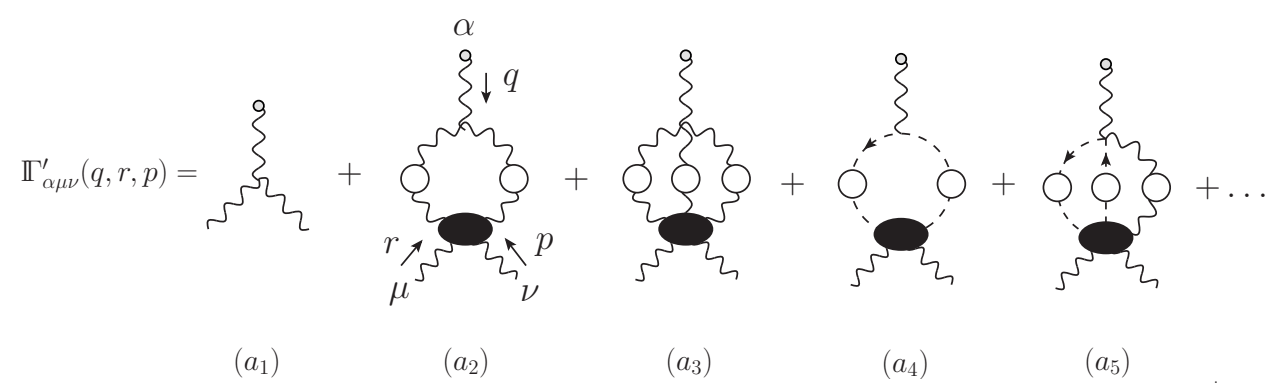

FIG. 2: The SDE for the BQQ vertex which connects a background gluon $(B)$ with two quantum gluons $(Q)$.

In general, when setting up the usual SDE for any vertex (see, for example, Fig. 2), a particular field (leg) is singled out, and is connected to the various multiparticle kernels through all elementary vertices of the theory involving this field (leg). The remaining legs enter into the various diagrams through the aforementioned multiparticle kernels (black circles in graphs $a_{2}-a_{5}$ in Fig. 2), or, in terms of the standard skeleton expansion, through fully-dressed vertices (instead of tree-level ones). For the case of the $B Q Q$ vertex that we 


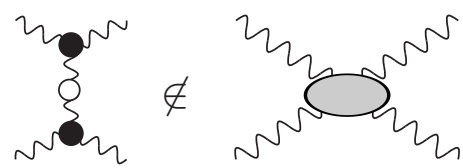

(a)

(B)

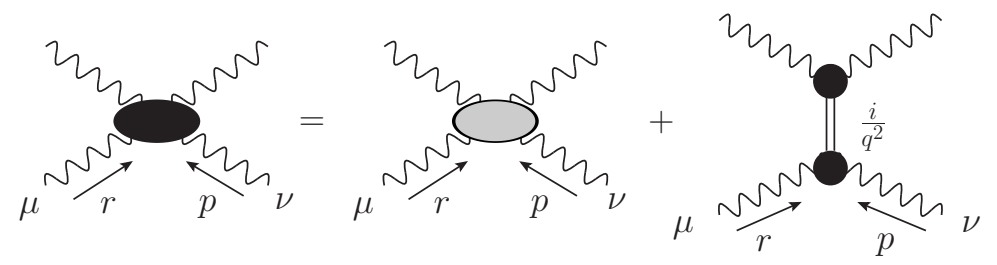

$\left(b_{1}\right)$

$\left(b_{2}\right)$

$\left(b_{3}\right)$

(C)

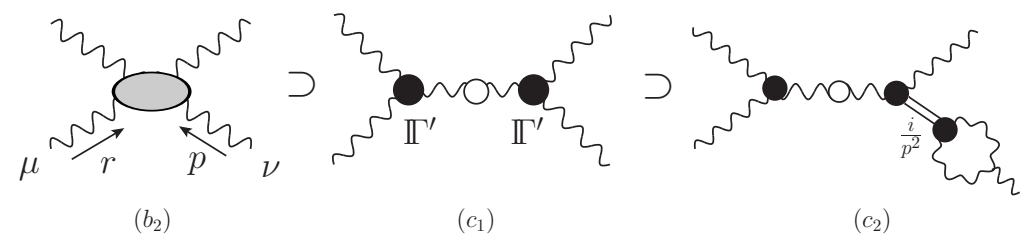

FIG. 3: (A) A diagram that does not belong to the standard kernel. (B) The gray kernel (regular part with respect to $q$, and the composite massless excitation in the $q$-channel. (C) The $R$ part of the vertex.

consider here [shown in Fig. 2], it is convenient (but not obligatory) to identify as the special leg the background gluon, carrying momentum $q$. Now, with the Schwinger mechanism turned off, the various multiparticle kernels appearing in the SDE for the $B Q Q$ vertex have a complicated skeleton expansion (not shown here), but their common characteristic is that they are one-particle-irreducible with respect to cuts in the direction of the momentum $q$; thus, a diagram such as the $(a)$ of Fig. 3 is explicitly excluded from the (gray) four-gluon kernel, and the same is true for all other kernels.

When the Schwinger mechanism is turned on, the structure of the kernels is modified by the presence of composite massless excitation, described by a propagator of the type $i / q^{2}$, as shown in Fig. 3. The sum of such dynamical terms, coming from all multiparticle kernels, shown in Fig. 4 constitutes a characteristic part of the vertex $V$, to be denoted by $U$ in Eq. (3.4), namely the part that contains at least a massless propagator $i / q^{2}$. The remaining parts of the vertex $V$, to be denoted by $R$ in Eq. (3.5), contain massless excitations in the other two channels, namely $1 / r^{2}$ and $1 / p^{2}$ (but no $1 / q^{2}$ ), and originate from graphs such as $\left(c_{2}\right)$ of Fig. 3. Indeed, note that the kernel $\left(b_{2}\right)$ is composed by an infinite number of 
diagrams, such as $\left(c_{1}\right)$, containing the full vertex $\mathbb{\Gamma}^{\prime}$; these graphs, in turn, will furnish terms proportional to $1 / r^{2}$ and $1 / p^{2}$ [e.g., graph $\left(c_{2}\right)$ ].

In order to study further the structure and properties of the vertex $V$, let us first define the full vertex $\mathcal{V}_{\alpha \mu \nu}^{a m n}(q, r, p)$, given by

$$
\mathcal{V}_{\alpha \mu \nu}^{a m n}(q, r, p)=g f^{a m n} V_{\alpha \mu \nu}(q, r, p)
$$

with $V_{\alpha \mu \nu}(q, r, p)$ satisfying Eq. (2.8). Using a general Lorentz basis, we have the following expansion for $V_{\alpha \mu \nu}(q, r, p)$ in terms of scalar form factors,

$$
\begin{aligned}
V_{\alpha \mu \nu}(q, r, p) & =V_{1} q_{\alpha} g_{\mu \nu}+V_{2} q_{\alpha} q_{\mu} q_{\nu}+V_{3} q_{\alpha} p_{\mu} p_{\nu}+V_{4} q_{\alpha} r_{\mu} q_{\nu}+V_{5} q_{\alpha} r_{\mu} p_{\nu} \\
& +V_{6} r_{\mu} g_{\alpha \nu}+V_{7} r_{\alpha} r_{\mu} r_{\nu}+V_{8} r_{\alpha} r_{\mu} p_{\nu}+V_{9} p_{\nu} g_{\alpha \mu}+V_{10} p_{\alpha} p_{\mu} p_{\nu}
\end{aligned}
$$

According to the arguments presented above, $V_{\alpha \mu \nu}(q, r, p)$ may be decomposed into

$$
V_{\alpha \mu \nu}(q, r, p)=U_{\alpha \mu \nu}(q, r, p)+R_{\alpha \mu \nu}(q, r, p)
$$

with

$$
U_{\alpha \mu \nu}(q, r, p)=q_{\alpha}\left(V_{1} g_{\mu \nu}+V_{2} q_{\mu} q_{\nu}+V_{3} p_{\mu} p_{\nu}+V_{4} r_{\mu} q_{\nu}+V_{5} r_{\mu} p_{\nu}\right)
$$

and

$$
R_{\alpha \mu \nu}(q, r, p)=\left(V_{6} g_{\alpha \nu}+V_{7} r_{\alpha} r_{\nu}+\frac{V_{8}}{2} r_{\alpha} p_{\nu}\right) r_{\mu}+\left(\frac{V_{8}}{2} r_{\alpha} r_{\mu}+V_{9} g_{\alpha \mu}+V_{10} p_{\alpha} p_{\mu}\right) p_{\nu}
$$

All form-factors of $U$ (namely $V_{1}-V_{5}$ ) must contain a pole $1 / q^{2}$, while some of them may contain, in addition, $1 / r^{2}$ and $1 / p^{2}$ poles. On the other hand, none of the form-factors of $R$ (namely $V_{6}-V_{10}$ ) contains $1 / q^{2}$ poles, but only $1 / r^{2}$ and $1 / p^{2}$ poles.

In what follows we will focus on $U_{\alpha \mu \nu}(q, r, p)$, which contains the explicit $q$-channel massless excitation, since this is the relevant channel in the SDE of the gluon propagator, where $V_{\alpha \mu \nu}(q, r, p)$ will be eventually inserted [graph $\left(a_{1}\right)$ in Fig. 1]. In fact, with the two internal gluons of diagram $\left(a_{1}\right)$ in the Landau gauge, we have that

$$
\begin{aligned}
P^{\mu^{\prime} \mu}(r) P^{\nu^{\prime} \nu}(p) V_{\alpha \mu \nu}(q, r, p) & =P^{\mu^{\prime} \mu}(r) P^{\nu^{\prime} \nu}(p) U_{\alpha \mu \nu}(q, r, p) \\
& =P^{\mu^{\prime} \mu}(r) P^{\nu^{\prime} \nu}(p) q_{\alpha}\left[V_{1}(q, r, p) g_{\mu \nu}+V_{2}(q, r, p) q_{\mu} q_{\nu}\right]
\end{aligned}
$$

so that the only relevant form factors are $V_{1}$ and $V_{2}$. 
(A)

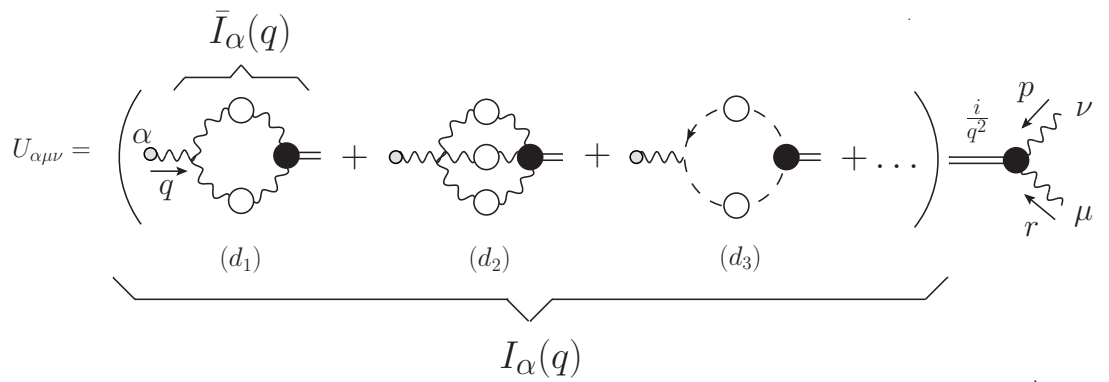

(B)

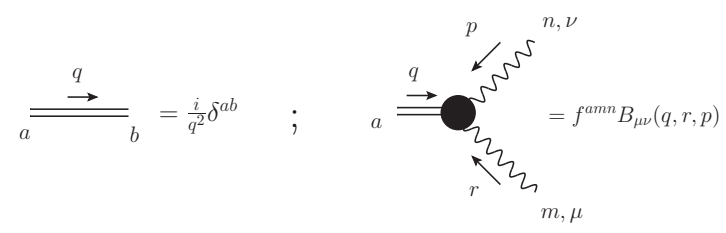

(C)

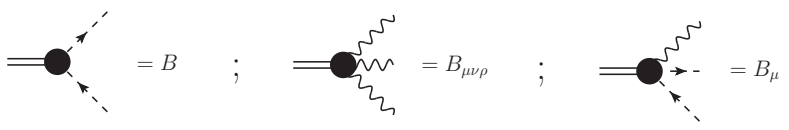

$\left(a_{1}\right)$

$\left(a_{2}\right)$

$\left(a_{3}\right)$

FIG. 4: (A) The vertex $U$ is composed of three main ingredients: the transition amplitude, $I_{\alpha}$, which mixes the gluon with a massless excitation, the propagator of the massless excitation, and the (massless excitation)-(gluon)-(gluon) vertex. (B) The Feynman rules (with color factors included) for (i) the propagator of the massless excitation and (ii) the "proper vertex function", or, "bound-state wave function", $B_{\mu \nu}$. (C) The various $B_{\{\ldots\}}$ appearing in Eq. (3.14).

At this point we can make the nonperturbative pole manifest, and cast $U_{\alpha \mu \nu}(q, r, p)$ in the form of Fig. 4, by setting

$$
U_{\alpha \mu \nu}(q, r, p)=I_{\alpha}(q)\left(\frac{i}{q^{2}}\right) B_{\mu \nu}(q, r, p),
$$

where the nonperturbative quantity

$$
B_{\mu \nu}(q, r, p)=B_{1} g_{\mu \nu}+B_{2} q_{\mu} q_{\nu}+B_{3} p_{\mu} p_{\nu}+B_{4} r_{\mu} q_{\nu}+B_{5} r_{\mu} p_{\nu}
$$

is the effective vertex (or "proper vertex function" [19]) describing the interaction between the massless excitation and two gluons. In the standard language used in bound-state physics, $B_{\mu \nu}(q, r, p)$ represents the "bound-state wave function" (or "BS wave function") of the two-gluon bound-state shown in $\left(b_{3}\right)$ of Fig. 3, as we will see in Section IV, $B_{\mu \nu}$ satisfies a (homogeneous) BSE. In addition, $i / q^{2}$ is the propagator of the scalar massless excitation. Finally, $I_{\alpha}(q)$ is the (nonperturbative) transition amplitude introduced in Fig. 4, allowing 
the mixing between a gluon and the massless excitation. Note that this latter function is universal, in the sense that it enters not only in the pole part $V$ associated with the threegluon vertex, but rather in all possible such pole parts associated with all other vertices, such as the four-gluon vertex, the gluon-ghost-ghost vertex, etc (see panel C in Fig. (4)).

Evidently, by Lorentz invariance,

$$
I_{\alpha}(q)=q_{\alpha} I(q)
$$

and the scalar cofactor, to be referred to as the "transition function", is simply given by

$$
I(q)=\frac{q^{\alpha} I_{\alpha}(q)}{q^{2}}
$$

so that

$$
V_{j}(q, r, p)=I(q)\left(\frac{i}{q^{2}}\right) B_{j}(q, r, p) ; \quad j=1, \ldots, 5 .
$$

Note that, due to Bose symmetry (already at the level of $V$ ) with respect to the interchange $\mu \leftrightarrow \nu$ and $p \leftrightarrow r$, we must have

$$
B_{1,2}(q, r, p)=-B_{1,2}(q, p, r)
$$

which implies that

$$
B_{1,2}(0,-p, p)=0
$$

Finally, in principle, all other elementary vertices of the theory may also develop pole parts, which will play a role completely analogous to that of $V_{\alpha \mu \nu}$ in maintaining the corresponding STIs in the presence of a gluon mass. Specifically, in the absence of quarks, the remaining vertices are the gluon-ghost-ghost vertex, $\mathbb{\Gamma}_{\alpha}$, the four-gluon vertex $\mathbb{\Gamma}_{\alpha \mu \nu \rho}$, and the gluon-gluon-ghost-ghost vertex $\mathbb{\Gamma}_{\alpha \mu}$, which is particular to the PT-BFM formulation. The parts of their pole vertices containing the $1 / q^{2}$, denoted by $U_{\alpha}, U_{\alpha \mu \nu \rho}$, and $U_{\alpha \mu}$, respectively, will all assume the common form

$$
U_{\alpha\{\ldots\}}=I_{\alpha}\left(\frac{i}{q^{2}}\right) B_{\{\ldots\}},
$$

where the various $B_{\{\ldots\}}$ are shown in panel C of Fig. 4 .

\section{B. An exact relation}

The WI of Eq (2.14) furnishes an exact relation between the dynamical gluon mass, the transition amplitude at zero momentum transfer, and the form factor $B_{1}$. Specifically, 
contracting both sides of the WI with two transverse projectors, one obtains,

$$
P^{\mu^{\prime} \mu}(r) P^{\nu^{\prime} \nu}(p) q^{\alpha} V_{\alpha \mu \nu}(q, r, p)=\left[m^{2}(r)-m^{2}(p)\right] P_{\sigma}^{\mu^{\prime}}(r) P^{\sigma \nu^{\prime}}(p)
$$

On the other hand, contracting the full expansion of the vertex (3.2) by these transverse projectors and then contracting the result with the momentum of the background leg, we get

$$
q^{\alpha} P^{\mu^{\prime} \mu}(r) P^{\nu^{\prime} \nu}(p) V_{\alpha \mu \nu}(q, r, p)=i I(q)\left[B_{1} g_{\mu \nu}+B_{2} q_{\mu} q_{\nu}\right] P^{\mu^{\prime} \mu}(r) P^{\nu^{\prime} \nu}(p)
$$

where the relation of Eq (3.11) has been used. Thus, equating both results, one arrives at

$$
i I(q) B_{1}(q, r, p)=m^{2}(r)-m^{2}(p), \quad B_{2}(q, r, p)=0 .
$$

The above relations, together with those of Eq. (3.11), determine exactly the form factors $V_{1}$ and $V_{2}$ of the vertex $V_{\alpha \mu \nu}$, namely

$$
V_{1}(q, r, p)=\frac{m^{2}(r)-m^{2}(p)}{q^{2}}, \quad V_{2}(q, r, p)=0 .
$$

We will now carry out the Taylor expansion of both sides of Eq (3.17) in the limit $q \rightarrow 0$. To that end, let consider the Taylor expansion of a function $f(q, r, p)$ around $q=0$ (and $r=-p)$. In general we have

$$
f(q,-p-q, p)=f(-p, p)+\left[2(q \cdot p)+q^{2}\right] f^{\prime}(-p, p)+2(q \cdot p)^{2} f^{\prime \prime}(-p, p)+\mathcal{O}\left(q^{3}\right)
$$

where the prime denotes differentiation with respect to $(p+q)^{2}$ and subsequently taking the limit $q \rightarrow 0$, i.e.

$$
f^{\prime}(-p, p) \equiv \lim _{q \rightarrow 0}\left\{\frac{\partial f(q,-p-q, p)}{\partial(p+q)^{2}}\right\} .
$$

Now, if the function is antisymmetric under $p \leftrightarrow r$, as happens with the form factors $B_{1,2}$, then $f(-p, p)=0$; thus, for the case of the form factors in question, the Taylor expansion is $(i=1,2)$

$$
B_{i}(q,-p-q, p)=\left[2(q \cdot p)+q^{2}\right] B_{i}^{\prime}(-p, p)+2(q \cdot p)^{2} B_{i}^{\prime \prime}(-p, p)+\mathcal{O}\left(q^{3}\right)
$$

Using Eq (3.21), and the corresponding expansion for the rhs,

$$
m^{2}(r)-m^{2}(p)=m^{2}(q+p)-m^{2}(p)=2(q \cdot p)\left[m^{2}(p)\right]^{\prime}+\mathcal{O}\left(q^{2}\right)
$$

assuming that the $I(0)$ is finite, and equating the coefficients in front of $(q \cdot p)$, we arrive at (Minkowski space)

$$
\left[m^{2}(p)\right]^{\prime}=i I(0) B_{1}^{\prime}(p)
$$


We emphasize that this is an exact relation, whose derivation relies only on the WI and Bosesymmetry that $V_{\alpha \mu \nu}(q, r, p)$ satisfies, as captured by Eq. (2.14) and Eq. (3.13), respectively. The Euclidean version of Eq. (3.23) is given in Eq. (5.1).

\section{C. "One-loop dressed" approximation for the transition function}

We will next approximate the transition amplitude $I_{\alpha}(q)$, connecting the gluon with the massless excitation, by considering only diagram $\left(d_{1}\right)$ in Fig. 4, corresponding to the gluonic "one-loop dressed" approximation; we will denote the resulting expression by $\bar{I}_{\alpha}(q)$.

In the Landau gauge, $\bar{I}_{\alpha}(q)$ is given by

$$
\bar{I}_{\alpha}(q)=\frac{1}{2} C_{A} \int_{k} \Delta(k) \Delta(k+q) \Gamma_{\alpha \beta \lambda} P^{\lambda \mu}(k) P^{\beta \nu}(k+q) B_{\mu \nu}(-q,-k, k+q),
$$

where the origin of the factor $1 / 2$ is combinatoric, and $\Gamma_{\alpha \beta \lambda}$ is the standard three-gluon vertex at tree-level,

$$
\Gamma_{\alpha \mu \nu}(q, r, p)=g_{\mu \nu}(r-p)_{\alpha}+g_{\alpha \nu}(p-q)_{\mu}+g_{\alpha \mu}(q-r)_{\nu}
$$

To determine the corresponding transition function from Eq. (3.10), use that

$$
q^{\alpha} \Gamma_{\alpha \beta \lambda}(q,-k-q, k)=\left[k^{2}-(k+q)^{2}\right] g_{\beta \lambda}+\left[(k+q)_{\beta}(k+q)_{\lambda}-k_{\beta} k_{\lambda}\right]
$$

to write

$$
\bar{I}(q)=-\frac{C_{A}}{2 q^{2}} \int_{k}\left[k^{2}-(k+q)^{2}\right] \Delta(k) \Delta(q+k) P_{\beta}^{\mu}(k) P^{\beta \nu}(k+q) B_{\nu \mu}(-q, k+q,-k) .
$$

In the last step we have used the property of Eq. (3.12) in order to interchange the arguments of $B_{\nu \mu}$, so that the Taylor expansion of Eq. (3.21) may be applied directly; this accounts for the additional minus sign. Then, after the shift $k+q \rightarrow k$, and further use of Eq. (3.12), $\bar{I}(q)$ becomes

$$
\bar{I}(q)=-\frac{C_{A}}{q^{2}} \int_{k} k^{2} \Delta(k) \Delta(k+q) P_{\beta}^{\mu}(k) P^{\beta \nu}(k+q)\left[B_{1} g_{\mu \nu}+B_{2} q_{\mu} q_{\nu}\right] .
$$

To obtain the limit of $\bar{I}(q)$ as $q^{2} \rightarrow 0$, we will employ Eq. (3.21) for $B_{1}$ and $B_{2}$, as well as

$$
\Delta(k+q)=\Delta(k)+\left[2(q \cdot k)+q^{2}\right] \Delta^{\prime}(k)+2(q \cdot k)^{2} \Delta^{\prime \prime}(k)+\mathcal{O}\left(q^{3}\right) .
$$


Observe that only the zeroth order term of $P_{\mu \nu}(k+q)$, namely $P_{\mu \nu}(k)$, contributes in this expansion. Then, using spherical coordinates to write $(q \cdot k)^{2}=q^{2} k^{2} \cos ^{2} \theta$, and the integral

$$
\int_{k} f(k) \cos ^{2} \theta=\frac{1}{d} \int_{k} f(k)
$$

the $\bar{I}(q)$ in Eq. (3.28) becomes in the limit $q^{2} \rightarrow 0($ in $d=4)$

$$
\bar{I}(0)=-3 C_{A}\left\{\int_{k} k^{2} \Delta^{2}(k) B_{1}^{\prime}(k)+\frac{1}{2} \int_{k} k^{4} \frac{\partial}{\partial k^{2}}\left[\Delta^{2}(k) B_{1}^{\prime}(k)\right]\right\} .
$$

Then, partial integration yields

$$
\int_{k} k^{4} \frac{\partial}{\partial k^{2}}\left[\Delta^{2}(k) B_{1}^{\prime}(k)\right]=-3 \int_{k} k^{2} \Delta^{2}(k) B_{1}^{\prime}(k),
$$

and finally one arrives at (Minkowski space)

$$
\bar{I}(0)=\frac{3}{2} C_{A} \int_{k} k^{2} \Delta^{2}(k) B_{1}^{\prime}(k) .
$$

The Euclidean version of this equation, Eq. (5.3), will be used in Section V.

We end this subsection with a comment on the dimensionality of the various form factors. The vertex $V_{\alpha \mu \nu}$ has dimension $[m]$, and so $V_{1}, V_{2}$ and $V_{3}$ are dimensionless, while the

remaining form factors have dimension $[m]^{-2}$. The integral $\bar{I}(q)$ has the same dimension as $B_{1}$, and as a result, in order to keep $V_{1}$ dimensionless, $B_{1}$ must have dimensions of $[m]$.

\section{Relating the gluon mass to the transition function}

In this subsection we show how the vertex $V$ gives rise to a gluon mass when inserted into the corresponding SDE. We will restrict ourselves to the two diagrams shown in Fig. 1, and will finally express $m^{2}(0)$ exclusively in terms of $\bar{I}(0)$, which, in turn, depends on the existence of $B_{\mu \nu}$ through Eq. (3.33).

In the PT-BFM scheme, the SDE of the gluon propagator in the Landau gauge assumes the form

$$
\Delta^{-1}\left(q^{2}\right) P_{\mu \nu}(q)=\frac{q^{2} P_{\mu \nu}(q)+i \Pi_{\mu \nu}(q)}{\left[1+G\left(q^{2}\right)\right]^{2}} .
$$

The most straightforward way to relate the gluon mass to the transition function $\bar{I}$ is to identify, on both sides of (3.34), the co-factors of the tensorial structure $q_{\mu} q_{\nu} / q^{2}$ which survive the limit $q^{2} \rightarrow 0$, and then set them equal to each other. Making the usual identification (in Minkowski space) $\Delta^{-1}(0)=-m^{2}(0)$, it is clear that lhs of (3.34) furnishes simply

$$
\left.\operatorname{lhs}\right|_{\frac{q_{\mu} q_{\nu}}{q^{2}}}=m^{2}(0)
$$




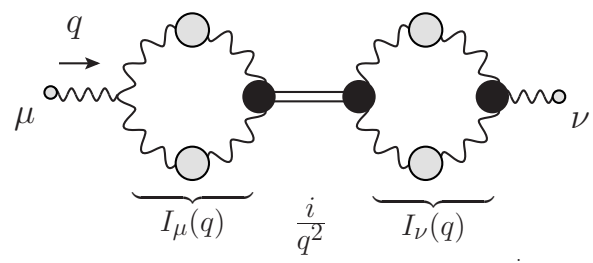

FIG. 5: The "squared" diagram.

It is relatively straightforward to recognize that the analogous contribution from the rhs comes from the standard "squared" diagram, shown in Fig. 5. Specifically, the starting expression is

$$
\Pi^{\mu \nu}(q)=\frac{1}{2} g^{2} C_{A} \int_{k} \Gamma_{\alpha \beta}^{\mu} P^{\alpha \rho}(k) P^{\beta \sigma}(k+q)[\mathbb{\Gamma}+V]_{\rho \sigma}^{\nu} \Delta(k) \Delta(k+q)+\cdots
$$

where, as explained earlier, the (all order) vertex $\mathbb{\Gamma}$ has been replaced by $\mathbb{\Gamma}+V$, and the ellipses denote terms that, in the kinematic limit considered, do not contribute to the specific structure of interest.

The relevant contribution originates from the part containing the vertex $V$, to be denoted by $\left.\Pi^{\mu \nu}(q)\right|_{V}$; it is represented by the diagram in Fig. 5. In particular, by virtue of Eq. (3.6), we have

$$
\begin{aligned}
\left.\Pi^{\mu \nu}(q)\right|_{V} & =\frac{1}{2} g^{2} C_{A} \int_{k} \Delta(q+k) \Delta(k) \Gamma_{\alpha \beta}^{\mu} P^{\alpha \rho}(k) P^{\beta \sigma}(k+q) U_{\rho \sigma}^{\nu} \\
& =g^{2}\left\{\frac{1}{2} C_{A} \int_{k} \Delta(q+k) \Delta(k) \Gamma_{\alpha \beta}^{\mu} P^{\alpha \rho}(k) P^{\beta \sigma}(k+q) B_{\rho \sigma}\right\}\left(\frac{i}{q^{2}}\right) \bar{I}_{\nu}(q) \\
& =i \frac{q^{\mu} q^{\nu}}{q^{2}} g^{2} \bar{I}^{2}(q),
\end{aligned}
$$

where in the second line we have used Eq. (3.7) $\left[\right.$ with $\left.I_{\nu}(q) \rightarrow \bar{I}_{\nu}(q)\right]$, and Eq. (3.24) in the third line.

Thus, using the fact that, since $L(0)=0$ [42], from the identity of Eq. (2.13) we have that $1+G(0)=F^{-1}(0)$, then the rhs of (3.34) becomes

$$
\left.\operatorname{rhs}\right|_{\frac{q_{\mu} q_{\nu}}{q^{2}}}=-g^{2} F^{2}(0) \bar{I}^{2}(0) .
$$

We next go to Euclidean space, following the usual rules, and noticing that, due to the change $\int_{k}=i \int_{k_{E}}$ we have $\bar{I}^{2}(0) \rightarrow-\bar{I}_{E}^{2}(0)$; so, equating (3.35) and (3.38) we obtain (suppressing the index " $\mathrm{E}$ ")

$$
m^{2}(0)=g^{2} F^{2}(0) \bar{I}^{2}(0) .
$$


Note that the $m^{2}(0)$ so obtained is positive-definite. We emphasize that the relation of Eq. (3.39) constitutes the (gluonic) "one-loop dressed" approximation of the complete relation; indeed, both the SDE used as starting point as well as the expression for $\bar{I}$ are precisely the corresponding "one-loop dressed" contributions, containing gluons (but not ghosts).

Finally, let us consider the exact relation [15]

$$
\widehat{m}^{2}\left(q^{2}\right)=\left[1+G\left(q^{2}\right)\right]^{2} m^{2}\left(q^{2}\right)
$$

expressing the dynamical mass $m\left(q^{2}\right)$ of the standard gluon propagator $\Delta\left(q^{2}\right)$ in terms of the corresponding mass, $\widehat{m}\left(q^{2}\right)$, of the PT-BFM gluon propagator [usually denoted by $\widehat{\Delta}\left(q^{2}\right)$ ] in the same gauge [in this case, in the Landau gauge]. At $q^{2}=0$ this relation reduces to $m^{2}(0)=\widehat{m}^{2}(0) F^{2}(0)$, so that Eq. (3.39) may be alternatively written as

$$
\widehat{m}^{2}(0)=g^{2} \bar{I}^{2}(0)
$$

Interestingly enough, when written in this form, the mass formula derived from our SDE analysis coincides with the one obtained for the photon mass in the Abelian model of Jackiw and Johnson (Eq. (2.12) in [18]). In addition, this last form facilitates the demonstration of the decoupling of the massless excitation from the on-shell four-gluon amplitude (see Section VI).

In principle, the analysis presented above may be extended to include the rest of the graphs contributing to the gluon SDE, invoking the corresponding pole parts of the remaining vertices; however, this lies beyond the scope of the present work.

\section{BS EQUATION FOR THE BOUND-STATE WAVE FUNCTION}

As has become clear in the previous section, the gauge boson (gluon) mass is inextricably connected to the existence of the quantity $B_{1}^{\prime}$. Indeed, if $B_{1}^{\prime}$ were to vanish, then, by virtue of (3.33) so would $\bar{I}(0)$, and therefore, through (3.39) we would obtain a vanishing $m^{2}(0)$. Thus, the existence of $B_{1}^{\prime}$ is of paramount importance for the mass generation mechanism envisaged here; essentially, the question boils down to whether or not the dynamical formation of a massless bound-state excitation of the type postulated above is possible. As is well-known, in order to establish the existence of such a bound state one must (i) derive the appropriate BSE for the corresponding bound-state wave function, $B_{\mu \nu}$, (or, in this case, its derivative), and (ii) find non-trivial solutions for this integral equation. 


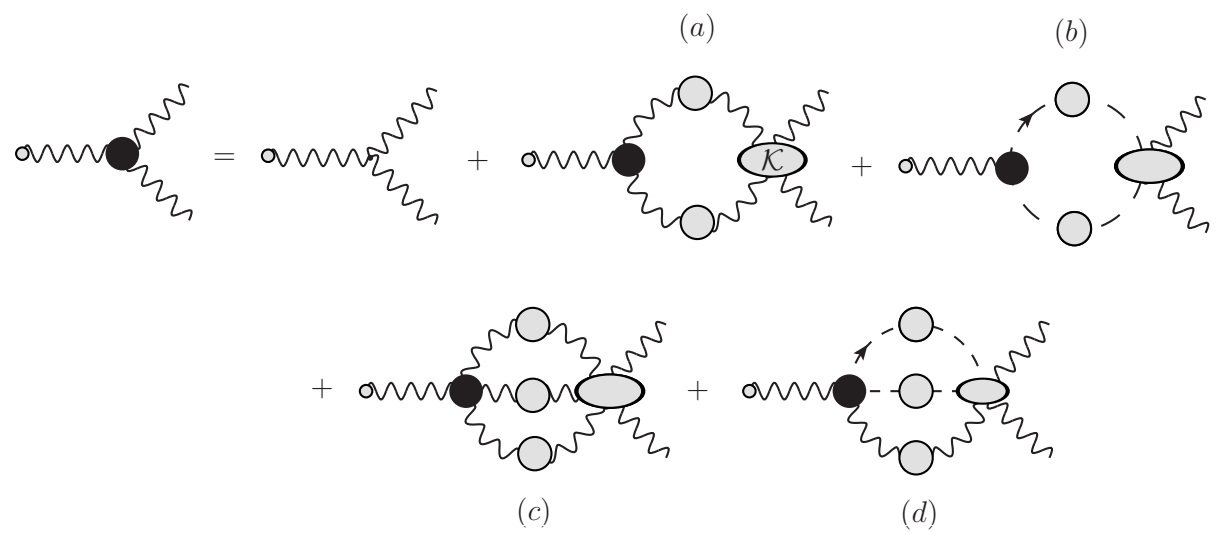

FIG. 6: The complete BSE for the full three gluon vertex $\mathbb{\Gamma}_{\alpha \mu \nu}^{\prime}(q, r, p)$.

To be sure, this dynamical equation will be derived under certain simplifying assumptions, which will be further refined in order to obtain numerical solutions. We emphasize, therefore, that the analysis presented here is meant to provide preliminary quantitative evidence for the realization of the dynamical scenario considered, but cannot be considered as a conclusive demonstration.

The starting point is the BSE for the vertex $\mathbb{\Gamma}_{\alpha \mu \nu}^{\prime}(q, r, p)$, shown in Fig. 6. Note that, unlike the corresponding SDE of Fig. 2, the vertices where the background gluon is entering (carrying momentum $q$ ) are now fully dressed. As a consequence, the corresponding multiparticle kernels appearing in Fig. 6 are different from those of the SDE, as shown in Fig. 7.
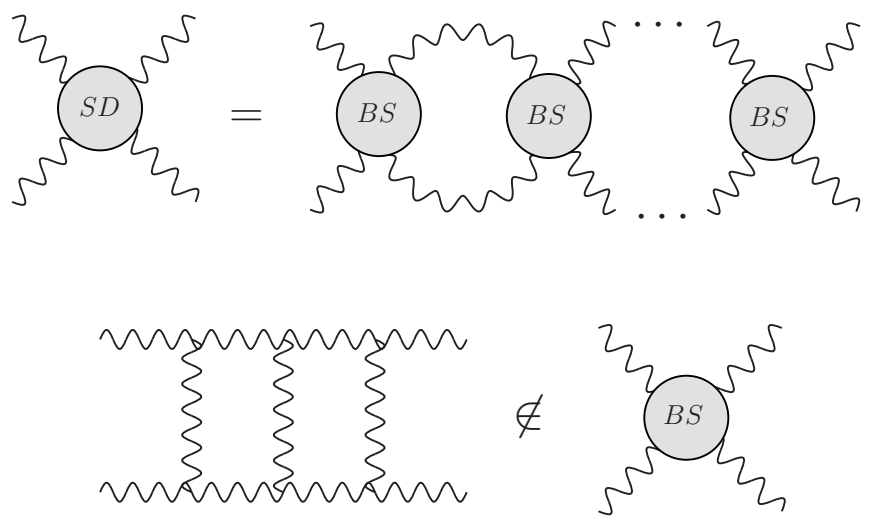

FIG. 7: (A) Schematic relation between the SDE and BSE kernels. (B) Example of a diagram not contained in the corresponding BSE kernel, in order to avoid over counting. 
(A)

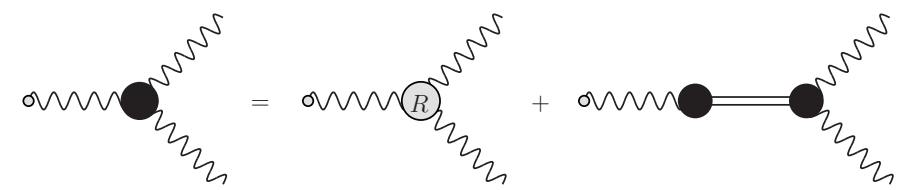

(B)

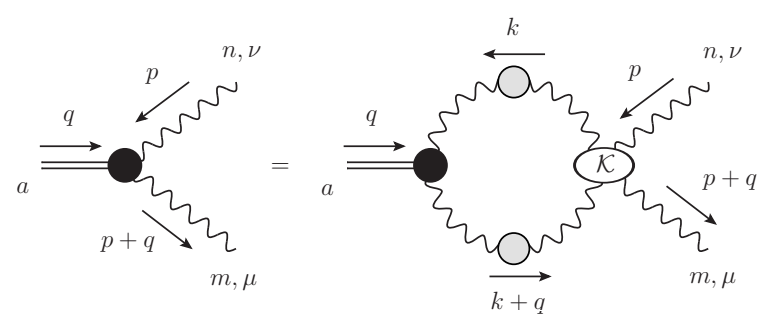

FIG. 8: (A) The separation of the vertex in regular and pole parts. (B) The BSE for the boundstate wave function $B_{\mu \nu}$.

The general methodology of how to isolate from the BSE shown in Fig. 6 the corresponding dynamical equation for the quantity $B_{\mu \nu}$ has been explained in [19, 22]. Specifically, one separates on both sides of the BSE equation each vertex (black circle) into two parts, a "regular" part and another containing a pole $1 / q^{2}$; this separation is shown schematically in Fig. 8. Then, the BSE for $B_{\mu \nu}(q, r, p)$ is obtained simply by equating the pole parts on both sides. Of course, for the case we consider the full implementation of this general procedure would lead to a very complicated structure, because, in principle, all fully dressed vertices appearing on the rhs of Fig. 6] may contain pole parts [i.e., not just the three-gluon vertex of (a) but also those in (b), (c), and (d)]. Thus, one would be led to an equation, whose lhs would consist of $B_{\mu \nu}$, but whose rhs would contain the $B_{\mu \nu}$ together with all other similar vertices, denoted by $B_{\{\ldots\}}$ in Eq. (3.14). Therefore, this equation must be supplemented by a set of analogous equations, obtained from the BSEs of all other vertices appearing on the rhs of Fig. 6 [i.e., those in (b), (c), (d) ]. So, if all vertices involved contain a pole part, one would arrive at a system of several coupled integral equations, containing complicated combinations of the numerous form factors composing these vertices (see, for example, Fig. 11 in [22]).

It is clear that for practical purposes the above procedure must be simplified to something more manageable. To that end, we will only consider graph (a) on the rhs of Fig. 6, thus reducing the problem to the treatment of a single integral equation. 

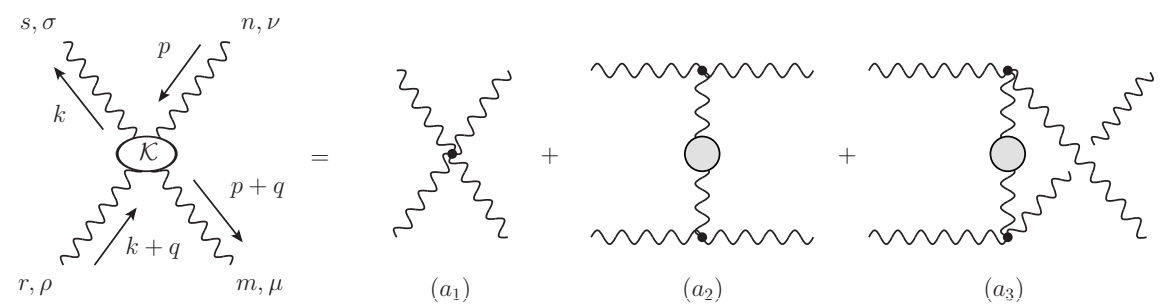

FIG. 9: The Feynman diagrams considered for the BS kernel. The interaction vertices are approximated by their tree level values, while the internal gluon propagators are fully dressed.

Specifically, the BSE for $B_{\mu \nu}$ is given by [see Fig. [6]

$$
B_{\mu \nu}^{a m n}=\int_{k} B_{\alpha \beta}^{a b c} \Delta_{b r}^{\alpha \rho}(k+q) \Delta_{c s}^{\beta \sigma}(k) \mathcal{K}_{\sigma \nu \mu \rho}^{s n m r} .
$$

In addition, we will approximate the four-gluon BS kernel $\mathcal{K}$ by the lowest-order set of diagrams shown in Fig. 9, where the vertices are bare, while the internal gluon propagators are fully dressed.

To proceed further, observe that the diagram $\left(a_{1}\right)$ does not contribute to the BSE, because the color structure of the tree-level four-gluon vertex vanishes when contracted with the color factor $f^{a b c}$ of the $B_{\alpha \beta}$. Diagrams $\left(a_{2}\right)$ and $\left(a_{3}\right)$ are equal, and are multiplied by a Bose symmetry factor of $1 / 2$. So, in this approximation, the BS kernel is given by

$$
\mathcal{K}_{\sigma \nu \mu \rho}^{s n m r}(-k, p,-p-q, k+q)=-i g^{2} f^{s n e} f^{e m r} \Gamma_{\sigma \gamma \nu}^{(0)} \Delta^{\gamma \lambda}(k-p) \Gamma_{\mu \lambda \rho}^{(0)},
$$

where $\Gamma^{(0)}$ is the tree-level value of the three gluon vertex. So, using this kernel and setting the gluon propagators in the Landau gauge, the BSE becomes

$$
B_{\mu \nu}=-2 \pi i \alpha_{s} C_{A} \int_{k} B_{\alpha \beta} \Delta(k+q) \Delta(k) \Delta(k-p) P^{\alpha \rho}(k+q) P^{\beta \sigma}(k) P^{\gamma \lambda}(k-p) \Gamma_{\sigma \gamma \nu}^{(0)} \Gamma_{\mu \lambda \rho}^{(0)},
$$

where we have cancelled out a color factor $f^{a b c}$ from both sides.

Let us focus on the lhs of Eq. (4.3). Using the Taylor expansion in Eq. (3.21), the fact that $B_{2}=0$ [see Eq. (3.17)], and multiplying by a transverse projector we obtain,

$$
P^{\mu \nu}(p) B_{\mu \nu}=6(q \cdot p) B_{1}^{\prime}(p)+\mathcal{O}\left(q^{2}\right)
$$

Next, let us denote by $[\mathrm{rhs}]_{\mu \nu}$ the rhs of Eq. (4.3). Inserting the bare value for the three gluon vertices, multiplying by the transverse projector, and using the Taylor expansions in Eq. (3.21) and (3.29), after standard manipulations one obtains the result

$$
P^{\mu \nu}(p)[\mathrm{rhs}]_{\mu \nu}=-4 \pi i \alpha_{s} C_{A}(q \cdot p) \int_{k} B_{1}^{\prime}(k) \Delta^{2}(k) \Delta(k-p) \mathcal{N}(p, k)+\mathcal{O}\left(q^{2}\right),
$$


where we have defined the kernel

$$
\mathcal{N}(p, k)=\frac{4(p \cdot k)\left[p^{2} k^{2}-(p \cdot k)^{2}\right]}{p^{4} k^{2}(k-p)^{2}}\left[8 p^{2} k^{2}-6(p k)\left(p^{2}+k^{2}\right)+3\left(p^{4}+k^{4}\right)+(p k)^{2}\right] .
$$

Thus, equating the lhs with the rhs, we derive the following BSE for the derivative of the form factor that appears in the mass relation Eq. (3.23),

$$
B_{1}^{\prime}(p)=-\frac{2 \pi i}{3} \alpha_{s} C_{A} \int_{k} B_{1}^{\prime}(k) \Delta^{2}(k) \Delta(k-p) \mathcal{N}(p, k) .
$$

Going to Euclidean space, we define

$$
x \equiv p^{2} ; \quad y \equiv k^{2} ; \quad z \equiv(p+k)^{2},
$$

and write the Euclidean integration measure in spherical coordinates,

$$
\int \frac{d^{4} k_{E}}{(2 \pi)^{4}}=\frac{1}{(2 \pi)^{3}} \int_{0}^{\infty} d y y \int_{0}^{\pi} d \theta \sin ^{2} \theta
$$

so that the BSE becomes

$$
\begin{aligned}
B_{1}^{\prime}(x)= & -\frac{\alpha_{s} C_{A}}{12 \pi^{2}} \int_{0}^{\infty} d y y B_{1}^{\prime}(y) \Delta^{2}(y) \\
& \sqrt{\frac{y}{x}} \int_{0}^{\pi} d \theta \sin ^{4} \theta \cos \theta\left[z+10(x+y)+\frac{1}{z}\left(x^{2}+y^{2}+10 x y\right)\right] \Delta(z) .
\end{aligned}
$$

In spherical coordinates we have that $z=x+y+2 \sqrt{x y} \cos \theta$. So, around $x=0$,

$$
\frac{1}{z}=\frac{1}{x+y}\left[1-2 \frac{\sqrt{x y}}{x+y} \cos \theta\right]
$$

and using the Taylor expansion for the gluon propagator $\Delta(z)$, the limit $x \rightarrow 0$ can be taken in the BSE, giving the value

$$
B_{1}^{\prime}(0)=\lim _{x \rightarrow 0} B_{1}^{\prime}(x)=-\frac{\alpha_{s} C_{A}}{8 \pi} \int_{0}^{\infty} d y y^{3} B_{1}^{\prime}(y) \Delta^{2}(y) \Delta^{\prime}(y) .
$$

Let us finally implement an additional simplification to Eq. (4.10), which will allow us to carry out the angular integration exactly, thus reducing the problem to the solution of a one-dimensional integral equation. Specifically, the simplification consists in approximating the gluon propagator $\Delta(z)$ appearing in the BSE of (4.10) [but not the $\Delta^{2}(y)$ ] by its tree level value, that is, $\Delta(z)=1 / z$. Then, with the aid of the angular integrals,

$$
\begin{aligned}
& \sqrt{\frac{y}{x}} \int_{0}^{\pi} d \theta \frac{\sin ^{4} \theta \cos \theta}{z}=\frac{\pi}{16 x}\left[\frac{y}{x^{2}}(y-2 x) \Theta(x-y)+\frac{x}{y^{2}}(x-2 y) \Theta(y-x)\right], \\
& \sqrt{\frac{y}{x}} \int_{0}^{\pi} d \theta \frac{\sin ^{4} \theta \cos \theta}{z^{2}}=-\frac{\pi}{4 x}\left[\frac{y}{x^{2}} \Theta(x-y)+\frac{x}{y^{2}} \Theta(y-x)\right],
\end{aligned}
$$


one brings Eq. (4.10) into the form

$$
\begin{aligned}
B_{1}^{\prime}(x)= & \frac{\alpha_{s} C_{A}}{24 \pi}\left\{\int_{0}^{x} d y B_{1}^{\prime}(y) \Delta^{2}(y) \frac{y^{2}}{x}\left(3+\frac{25}{4} \frac{y}{x}-\frac{3}{4} \frac{y^{2}}{x^{2}}\right)+\right. \\
& \left.+\int_{x}^{\infty} d y B_{1}^{\prime}(y) \Delta^{2}(y) y\left(3+\frac{25}{4} \frac{x}{y}-\frac{3}{4} \frac{x^{2}}{y^{2}}\right)\right\} .
\end{aligned}
$$

The limit $x \rightarrow 0$ of this equation is given by (the change of variable $y=t x$ may be found useful),

$$
B_{1}^{\prime}(0)=\frac{\alpha_{s} C_{A}}{8 \pi} \int_{0}^{\infty} d y y B_{1}^{\prime}(y) \Delta^{2}(y) .
$$

Note that this result coincides, as it should, with that obtained from Eq. (4.12) after setting $\Delta^{\prime}(y)=-1 / y^{2}$, namely the derivative of the tree-level propagator.

\section{NUMERICAL SOLUTIONS AND EXISTENCE OF A BOUND-STATE}

In this section we will carry out a detailed numerical analysis of the integral equation obtained in the previous section, namely Eq. (4.14).

First of all, let us point out that, despite appearances, the integral equation (4.14) is not linear in the unknown function $B_{1}^{\prime}(x)$. The non-linearity enters through the propagator $\Delta(y)$, which depends on the dynamical mass $m^{2}(y)$ through Eq. (2.6); as a result, and by virtue of Eq. (3.23), which, in Euclidean space reads

$$
\left[m^{2}(y)\right]^{\prime}=-I(0) B_{1}^{\prime}(y)
$$

it is clear that $\Delta(y)$ depends on $B_{1}^{\prime}(x)$ in a complicated way. Specifically, from the two aforementioned equations we have

$$
\begin{aligned}
\Delta^{-1}(y) & =y J(y)+m^{2}(y) \\
m^{2}(y) & =m^{2}(0)-I(0) \int_{0}^{y} d z B_{1}^{\prime}(z) .
\end{aligned}
$$

where $I(0)$ may be approximated by its "one-loop dressed" version $\bar{I}(0)$ given in (3.33), which in Euclidean space becomes

$$
\bar{I}(0)=\frac{3 C_{A}}{32 \pi^{2}} \int_{0}^{\infty} d y y^{2} \Delta^{2}(y) B_{1}^{\prime}(y) .
$$

Then, Eq. (4.14) must be solved together with the two additional relations given in Eq. (5.2), as a non-linear system. In addition, one may use Eq. (3.39), in order to obtain an 
(approximate) constraint for $I(0)$. Note also that Eq. (4.14), again due to Eq. (5.1), may be recast entirely in terms of $m^{2}(y)$ and its derivative.

For the purposes of the present work we will simplify somewhat the procedure described above. Specifically, we will present two different approaches, each one particularly suited for probing distinct features of Eq. (4.14) and the accompanying Eqs. (5.2). In particular, we will first study Eq. (4.14) in isolation, using simple Ansätze for $\Delta(y)$. The purpose of this study is to establish the existence of non-trivial solutions for $B_{1}^{\prime}$, study their dependence on the value of the strong coupling $\alpha_{s}$, and verify the asymptotic behavior predicted by Eq. (5.4). Of course, since $\Delta(y)$ at this level is treated as an "external" object, the homogeneous Eq. (4.14) becomes linear in $B_{1}^{\prime}$; as a result, given one solution we obtain a family of such solutions, through multiplication by any real constant. Then, as a second step, we will use the available lattice data for the gluon propagator $\Delta(y)$, in order to obtain the corresponding solution for $B_{1}^{\prime}$. Now, the linearity induced by treating $\Delta(y)$ as an external input will be resolved by resorting to Eq. (5.2) and Eq. (3.39); thus, out of the infinite family of solutions only one will be dynamically selected. These two approaches will be presented in subsections $\mathrm{VB}$ and $\mathrm{VC}$, while subsection $\mathrm{VA}$ deals with the asymptotic behavior of the solutions.

\section{A. Asymptotic behavior}

Before turning to the numerical treatment of Eq. (4.14), it is useful to study the behavior of the solutions for asymptotically large values of $x$. Setting $\Delta(y)=1 / y$, it is relatively straightforward to establish that the equation admits a power-law solution of the form $B_{1}^{\prime}(x)=A x^{b}$. Specifically, substituting this Ansatz into the first integral of Eq. (4.14), which is the dominant part for large $x$, and carrying out the integrations, one arrives at the following algebraic equation for $b$,

$$
\frac{24 \pi}{\alpha_{s} C_{A}}=\frac{3}{b+1}+\frac{25}{4(b+2)}-\frac{3}{4(b+3)},
$$

together with the restriction $b>-1$, imposed in order to assure convergence in the lower $(y=0)$ limit of integration. Setting $\lambda \equiv 24 \pi / \alpha_{s} C_{A}$, one arrives at the third-order equation

$$
4 \lambda b^{3}-(34-24 \lambda) b^{2}-(151-44 \lambda) b-141+24 \lambda=0
$$

that may be easily solved; the solution that satisfies $b>-1$ is shown in Fig. 10 as a function of $\alpha_{s}$. 


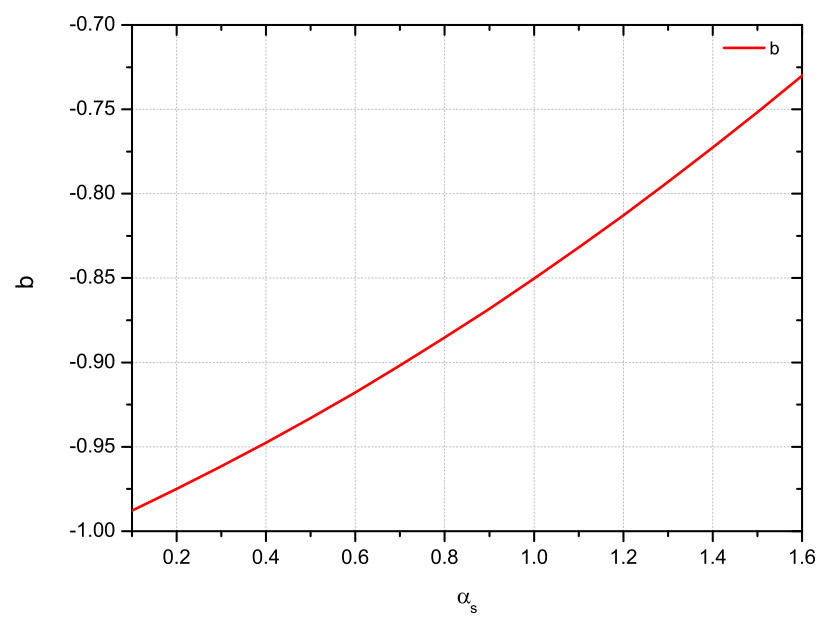

FIG. 10: The physically relevant solution of Eq. (5.5).

\section{B. The linearized case: solutions for various gluon propagators}

Next we discuss the numerical solutions for Eq. (4.14) for arbitrary values of $x$. Evidently, the main ingredient entering into its kernel is the nonperturbative gluon propagator, $\Delta(q)$. In order to explore the sensitivity of the solutions on the details of $\Delta(q)$, we will employ three infrared-finite forms, to be denoted by $\Delta_{1}(q), \Delta_{2}(q)$, and $\Delta_{3}(q)$, focusing on their differences in the intermediate and asymptotic regions of momenta.

Let us start with the simplest such propagator, namely a tree-level massive propagator of the form

$$
\Delta_{1}^{-1}\left(q^{2}\right)=q^{2}+m_{0}^{2}
$$

where $m_{0}^{2}$ is a hard mass, that will be treated as a free parameter. On the left panel of Fig. 11, the (blue) dotted curve represents $\Delta_{1}\left(q^{2}\right)$ for $m_{0}=376 \mathrm{MeV}$.

The second model is an improved version of the first, where we introduce the renormalization-group logarithm next to the momentum $q^{2}$, more specifically

$$
\Delta_{2}^{-1}\left(q^{2}\right)=q^{2}\left[1+\frac{13 C_{\mathrm{A}} g^{2}}{96 \pi^{2}} \ln \left(\frac{q^{2}+\rho m_{0}^{2}}{\mu^{2}}\right)\right]+m_{0}^{2} .
$$

where $\rho$ is an adjustable parameter varying in the range of $\rho \in[2,10]$. Notice that the hard mass $m_{0}^{2}$ appearing in the argument of the perturbative logarithm acts as an infrared cutoff; so, instead of the logarithm diverging at the Landau pole, it saturates at a finite value. The (black) dashed line represents the Eq. (5.7) when $\rho=16, m_{0}=376 \mathrm{MeV}$, and $\mu=4.3 \mathrm{GeV}$.

The third model is simply a physically motivated fit for the gluon propagator determined 

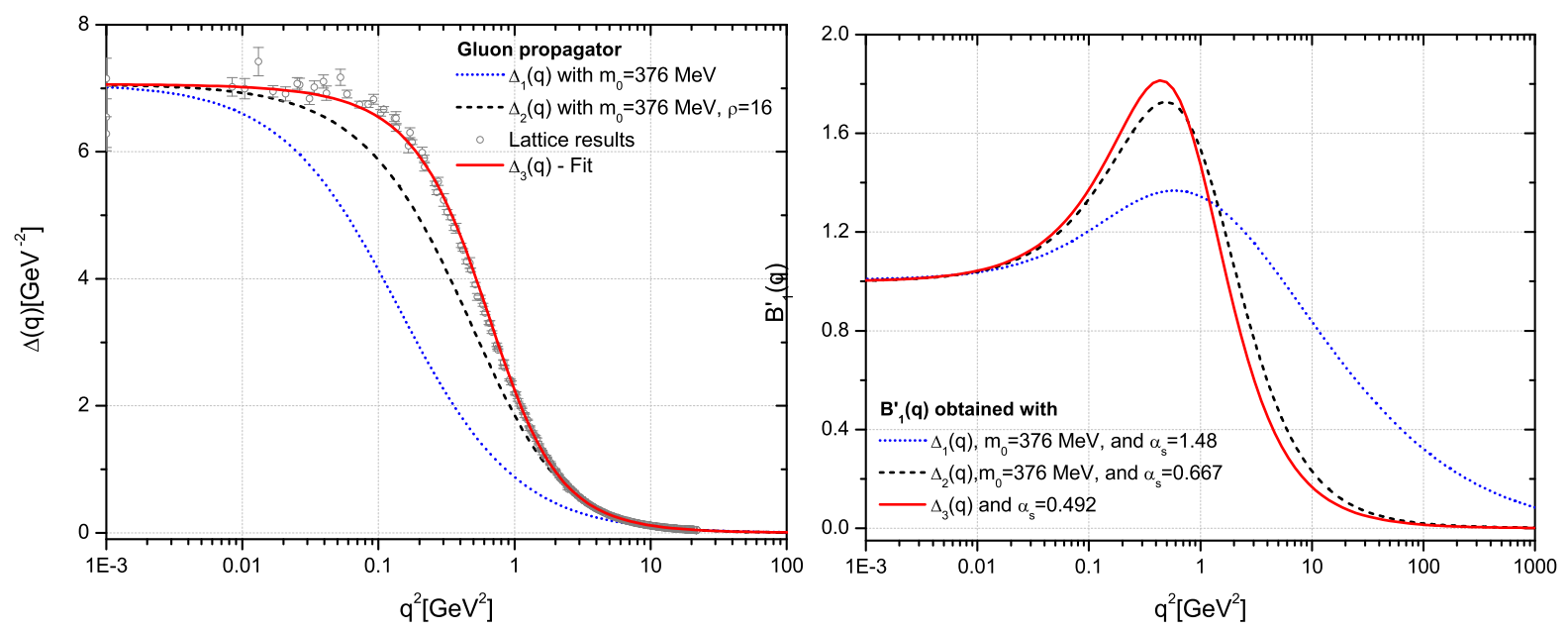

FIG. 11: Left panel: The three models for the gluon propagator as function of the momentum $q^{2}$. The (red) continuous line is the fit for the lattice gluon propagator given by Eq. (5.8) when $m=520 \mathrm{MeV}, g_{1}^{2}=5.68, \rho_{1}=8.55, \rho_{2}=1.91$, and $\mu=4.3 \mathrm{GeV}$; the (black) dashed line is the model of Eq. (5.7) with $\rho=16, \alpha_{s}=0.667$ and $m_{0}=376 \mathrm{MeV}$, while the (blue) dotted line represents the massive propagator of Eq. (5.6) when $m_{0}=376 \mathrm{MeV}$. Right panel: The corresponding solutions of Eq. (4.14) obtained with the gluon propagators shown on the left panel. The solutions for $B_{1}^{\prime}(q)$ are obtained when we fix the value of $\alpha_{s}=1.48, \alpha_{s}=0.667$, and $\alpha_{s}=0.492$ for $\Delta_{1}(q)$, $\Delta_{2}(q)$, and $\Delta_{3}(q)$, respectively.

by the large-volume lattice simulations of Ref. [6], and shown on the left panel of Fig. 11, The lattice data presented there correspond to a $S U(3)$ quenched lattice simulation, where $\Delta(q)$ is renormalized at $\mu=4.3 \mathrm{GeV}$. This gluon propagator can be accurately fitted by the expression (e.g.,[15])

$$
\Delta_{3}^{-1}\left(q^{2}\right)=m_{g}^{2}\left(q^{2}\right)+q^{2}\left[1+\frac{13 C_{\mathrm{A}} g_{1}^{2}}{96 \pi^{2}} \ln \left(\frac{q^{2}+\rho_{1} m_{g}^{2}\left(q^{2}\right)}{\mu^{2}}\right)\right],
$$

where $m_{g}^{2}\left(q^{2}\right)$ is a running mass given by

$$
m_{g}^{2}\left(q^{2}\right)=\frac{m^{4}}{q^{2}+\rho_{2} m^{2}}
$$

and the values of the fitting parameters are $m=520 \mathrm{MeV}, g_{1}^{2}=5.68, \rho_{1}=8.55$ and, $\rho_{2}=1.91$. On the left panel of Fig. 11, the (red) continuous line represents the fit for the lattice gluon propagator given by Eq. (5.8). Notice that, in all three cases, we have fixed the value of $\Delta^{-1}(0)=m_{0}^{2} \approx 0.14$. 


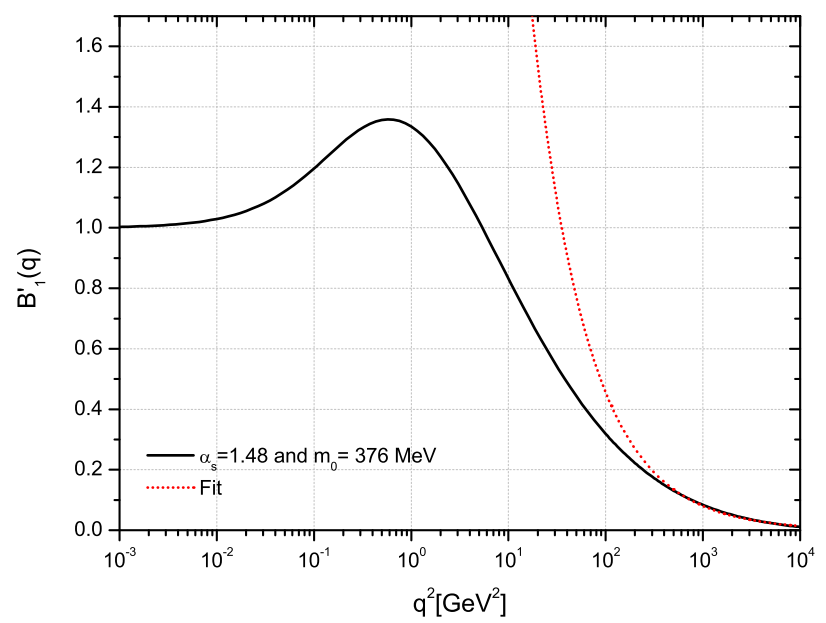

FIG. 12: The (black) continuous curve represents the solution obtained from Eq. (4.14) using the propagator $\Delta_{1}(q)$ of Eq. (5.6), with $\alpha_{s}=1.48$ and $m_{0}=376 \mathrm{MeV}$. The (red) dotted line is the best fit obtained for the asymptotic behavior of $B_{1}^{\prime}(x)$ given by $B_{1}^{\prime}(q)=A q^{2 b}$ with $A_{1}=14.80$ and $b=-0.756$. Notice that this value is in excellent agreement with the power found by the analytical determination, shown in Fig. 10.

Our main findings may be summarized as follows.

(i) In Fig. 11, right panel, we show the solutions of Eq. (4.14) obtained using as input the three propagators shown on the left panel. For the simple massive propagator of Eq. (5.6), a solution for $B_{1}^{\prime}(q)$ is found for $\alpha_{s}=1.48$; in the case of $\Delta_{2}(q)$ given by Eq. (5.7), a solution is obtained when $\alpha_{s}=0.667$, while for the lattice propagator $\Delta_{3}(q)$ of Eq. (5.8) a non-trivial solution is found when $\alpha_{s}=0.492$.

(ii) Note that, due to the fact that Eq. (4.14) is homogeneous and (effectively) linear, if $B_{1}^{\prime}(q)$ is a solution then the function $c B_{1}^{\prime}(q)$ is also a solution, for any real constant $c$. Therefore, the solutions shown on the right panel of Fig. 11 corresponds to a representative case of a family of possible solutions, where the constant $c$ was chosen such that $B_{1}^{\prime}(0)=1$.

(iii) Another interesting feature of the solutions of Eq. (4.14) is the dependence of the observed peak on the support of the gluon propagator in the intermediate region of momenta. Specifically, an increase of the support of the gluon propagator in the approximate range (0.3-1) GeV results in a more pronounced peak in $B_{1}^{\prime}(q)$.

(iv) In addition, observe that due to the presence of the perturbative logarithm in the expression for $\Delta_{2}(q)$ and $\Delta_{3}(q)$, the corresponding solutions $B_{1}^{\prime}(q)$ fall off in the ultraviolet region much faster than those obtained using the simple $\Delta_{1}(q)$ of Eq. (5.6). In order to 

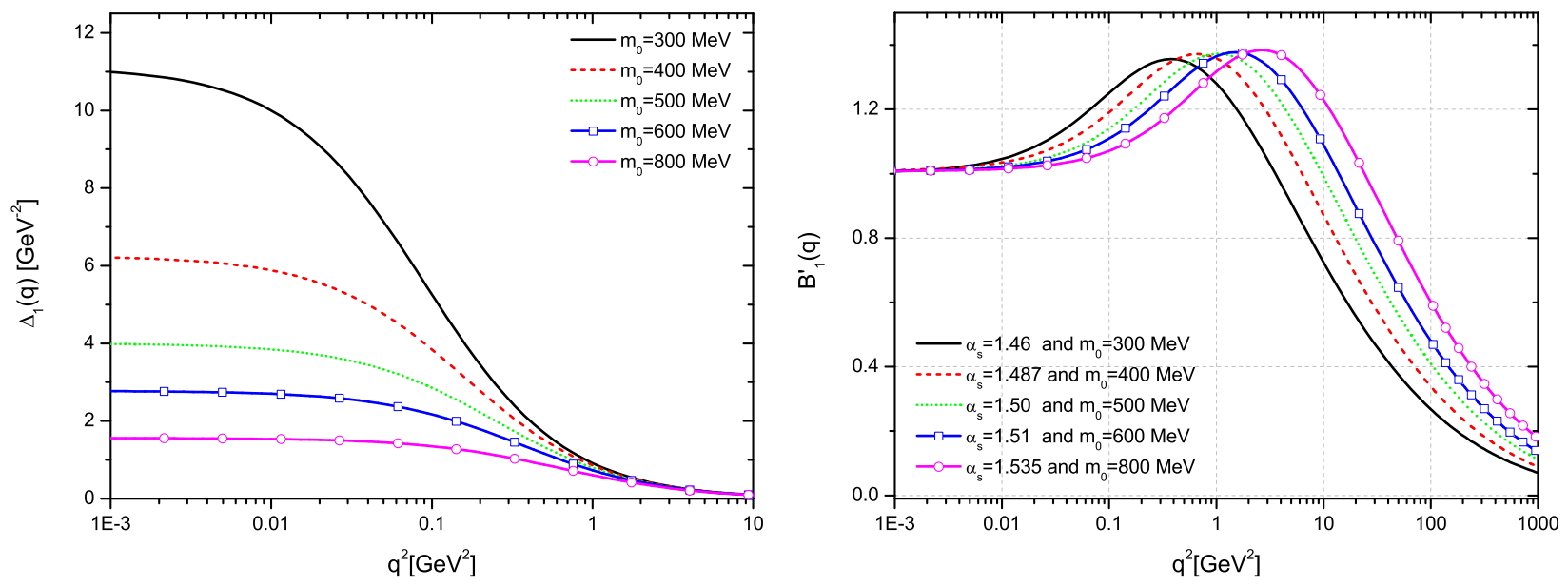

FIG. 13: Left panel: The behavior of the gluon propagator $\Delta_{1}(q)$, given by Eq. (5.6), for various values of $m_{0}$ in the range of $300-800 \mathrm{MeV}$. Right panel: The corresponding solutions for $B_{1}^{\prime}(q)$, obtained using the gluon propagators shown on the left panel. For each value of $m_{0}$, we found that the solution for $B_{1}^{\prime}(q)$ is obtained for a particular value of $\alpha_{s}$.

check whether the power-law asymptotic behavior, $B_{1}^{\prime}(q)=A q^{2 b}$, determined in our previous analysis, is in agreement with our numerical solution, we isolate in Fig. 12 the solution of $B_{1}^{\prime}(q)$ obtained with $\Delta_{1}(q)$ and $\alpha_{s}=1.48$ (black continuous curve) and compare it with the best fit obtained for large values of $q^{2}$ (red dotted curve). Indeed, the asymptotic tail of $B_{1}^{\prime}(q)$ falls off as power law of the type $B_{1}^{\prime}(q)=A q^{2 b}$ with $A=14.80$ and $b=-0.756$. Notice that the value of $b$ obtained from the fit is in perfect agreement with values obtained from Eq. (5.5), shown in Fig. 10,

(v) On the left panel of Fig. 13 we plot $\Delta_{1}(q)$, given by Eq. (5.6), for different values of $m_{0}$ in the range of $300-800 \mathrm{MeV}$. In order to determine how the solutions are modified when one varies the value of $m_{0}$, we show on the right panel of Fig. 13$]$ the various $B_{1}^{\prime}(q)$, all of them normalized at $B_{1}^{\prime}(0)=1$. As we can see, the solutions display the same qualitative behavior; however, for each $m_{0}$, the non-trivial solution is obtained for a different value of $\alpha_{s}$. In fact, as the values of $m_{0}$ increase, so do the values of $\alpha_{s}$ needed for obtaining a solution; the exact dependence of $\alpha_{s}$ on $m_{0}^{2}$ is shown in Fig. 14,

(vi) Next, we study how size variations in the intermediate region of the gluon propagator change the values of $\alpha_{s}$ needed in order to obtain non-trivial solutions from Eq. (4.14). To address this point systematically, we employ the gluon propagator $\Delta_{2}(q)$ of Eq. (5.7), varying the parameter $\rho$ in the range of $\rho \in[2,16]$, keeping fixed $m_{0}=367 \mathrm{MeV}$, as shown on the left 


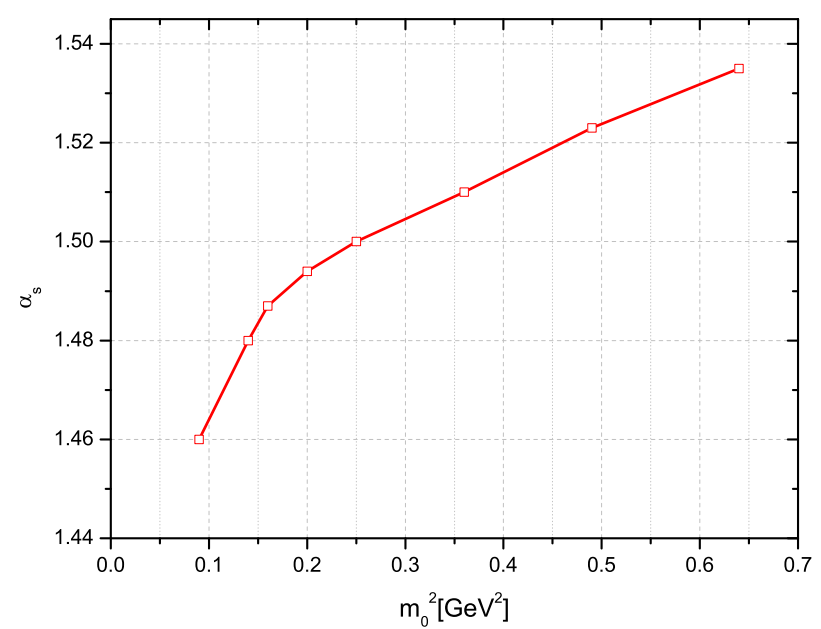

FIG. 14: The values of $\alpha_{s}$ furnishing non-trivial solutions to Eq. (4.14) as we vary $m_{0}$ in the $\Delta_{1}(q)$ of Eq. (5.6).

panel of Fig. 15, the corresponding $B_{1}^{\prime}(q)$ for each value of $\rho$ are plotted on the right panel. Evidently, decreasing $\rho$ increases the support of the gluon propagator in the intermediate region, and, as a result, one needs smaller value of $\alpha_{s}$ in order to obtain solutions for $B_{1}^{\prime}(q)$. This last property is better seen in Fig. 16, where we present the values of $\alpha_{s}$ needed to solve Eq. (4.14) as one varies $\rho$ in $\Delta_{2}(q)$.
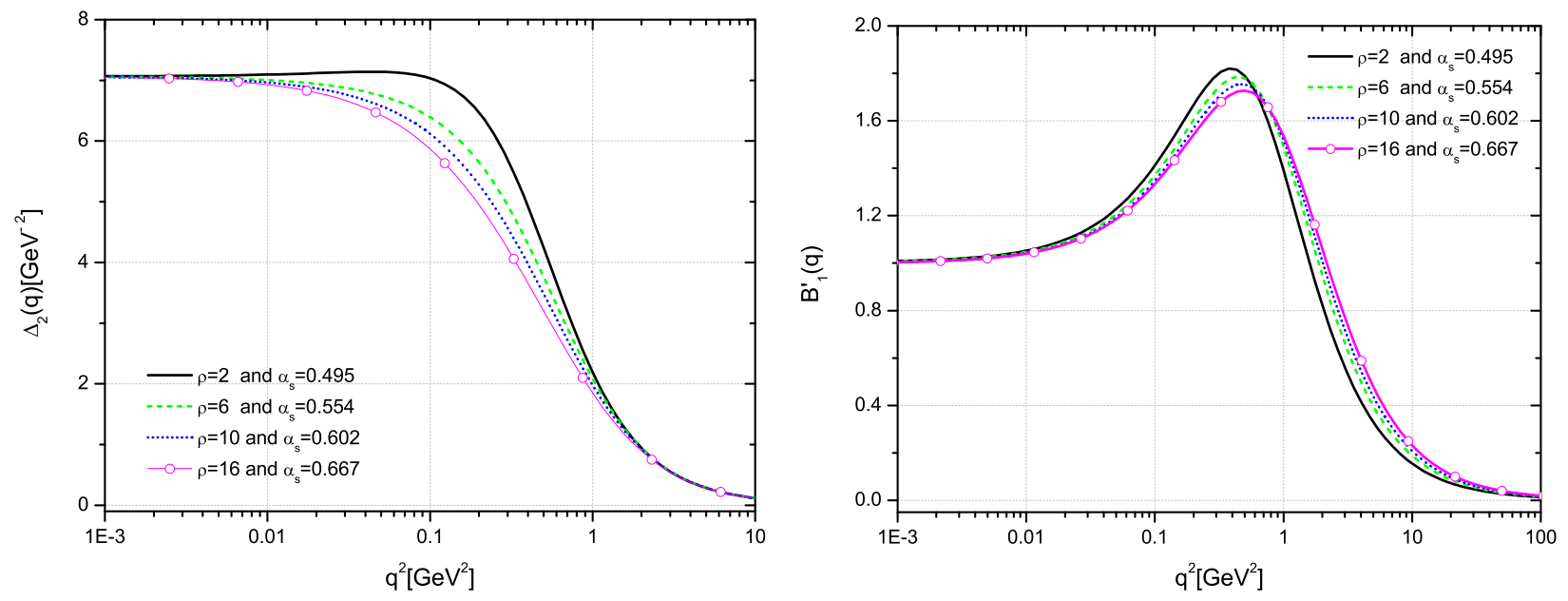

FIG. 15: Left panel: The behavior of the gluon propagator, $\Delta_{2}(q)$, given by Eq. (5.7), when the value of $m_{0}=367 \mathrm{MeV}$ is fixed, and $\rho$ varies in the range 2-16. Right panel: The corresponding solutions for $B_{1}^{\prime}(q)$ obtained with the gluon propagators shown on the left panel. To each value of $\rho$ corresponds a specific value of $\alpha_{s}$ that yields a solution $B_{1}^{\prime}(q)$. 


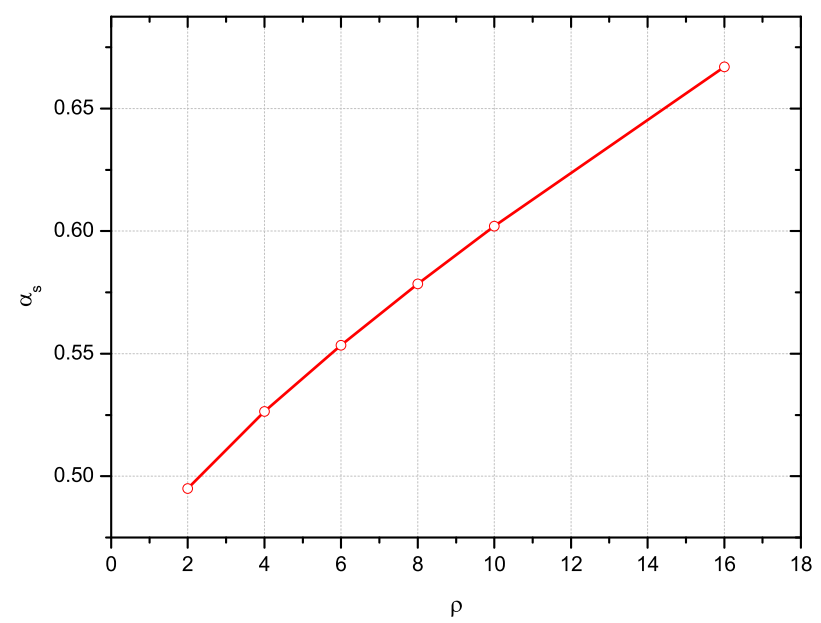

FIG. 16: The values of $\alpha_{s}$ for which we obtain non-trivial solutions to Eq. (4.14) as we vary $\rho$ in $\Delta_{2}(q)$ of Eq. (5.7).

\section{Non-linear treatment: uniqueness of $B_{1}^{\prime}(x)$ and $m^{2}(x)$}

In the previous subsection we have practically solved Eq. (4.14) in isolation, in the sense that we have not used the supplementary conditions of Eq. (15.2), and have treated $\Delta(q)$ as an external independent quantity. As a result, the homogeneous Eq. (4.14) was effectively linearized, giving rise to families of solutions $c B_{1}^{\prime}(x)$, parametrized by the value of $c$. In this subsection we will restore the non-linearity of Eq. (4.14); as a result, the arbitrariness in the value of $c$ is completely eliminated, and one obtains a single expression for $B_{1}^{\prime}(x)$ and $m^{2}(x)$, for a unique value of $\alpha_{s}$.

The way a unique solution for $B_{1}^{\prime}(x)$ is singled out [i.e., a value for $c$ is dynamically chosen] is by combining Eq. (3.39) and Eq. (5.3); specifically, we will require that the value for $\bar{I}(0)$ obtained from the former equation coincides with that obtained from the latter, namely that

$$
\sqrt{\Delta^{-1}(0) / 4 \pi \alpha_{s} F^{2}(0)}=\frac{3 C_{A}}{32 \pi^{2}} \int_{0}^{\infty} d y y^{2} \Delta^{2}(y) B_{1}^{\prime}(y)
$$

Now, the lhs of (5.10) is fixed, because, as mentioned in the previous subsection, we must have $\alpha_{s}=0.492$ in order for Eq. (4.14) to have solutions for this particular (lattice) propagator as input, while $\Delta^{-1}(0)$ and $F(0)$ are fixed from the lattice. Specifically, the $S U(3)$ large-volume lattice simulations of Ref. [6] yield $\Delta^{-1} \approx 0.141$ (see Fig. 11) and $F(0) \approx 2.76$ (see Fig. 17). Then, the integral on the rhs (5.10) must match the value of the lhs, and this can only happen for one particular member of the family $c B_{1}^{\prime}(x)$.

In Fig. 18, we show the solution for $B_{1}^{\prime}(x)$, which satisfies the constraints imposed on the 


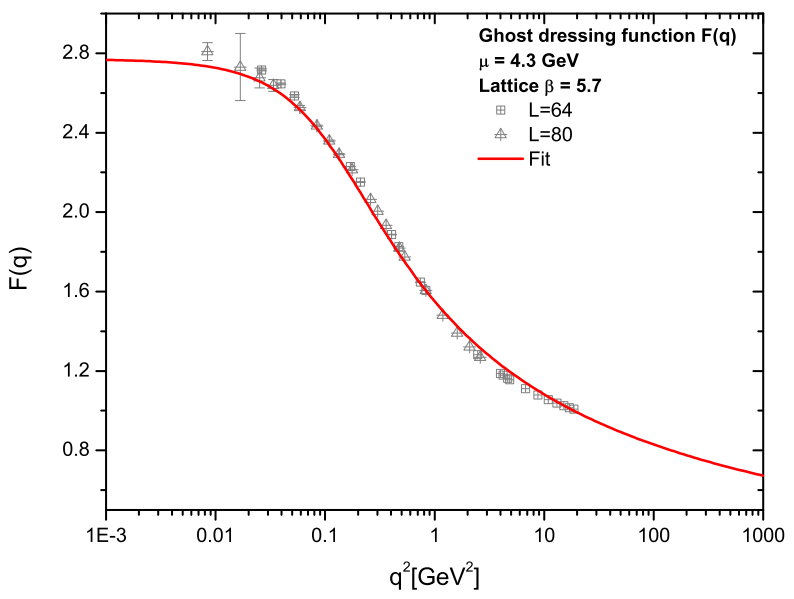

FIG. 17: Lattice results [6] for the ghost dressing function, $F(q)$, renormalized at $\mu=4.3 \mathrm{GeV}$. Notice that $F(0) \approx 2.76$.

value of $I(0)$, obtained when $\alpha_{s}=0.492$ and $B_{1}^{\prime}(0)=0.086$.

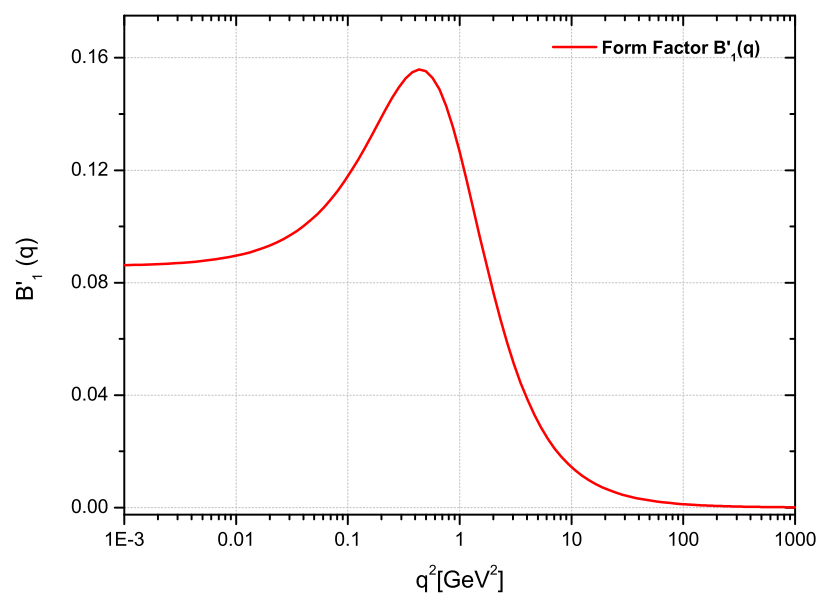

FIG. 18: The numerical solution $B_{1}^{\prime}(q)$ obtained from Eq. (4.14), under the constraints imposed by Eqs. (3.39) and (3.33), and with $\alpha_{s}=0.492$.

Once the unique solution $B_{1}^{\prime}(q)$ has been determined, one may use Eq. (5.2) to determine the behavior of the squared gluon mass $m^{2}(x)$. Integrating numerically $B_{1}^{\prime}(q)$ and fixing $m(0)=0.14$, we obtain the result shown in Fig. 19.

Evidently, the function $m^{2}\left(q^{2}\right)$ displays a plateau in the deep infrared, and then decreases sufficiently fast in the ultraviolet region, as expected on general grounds [10, 14, 15]. 


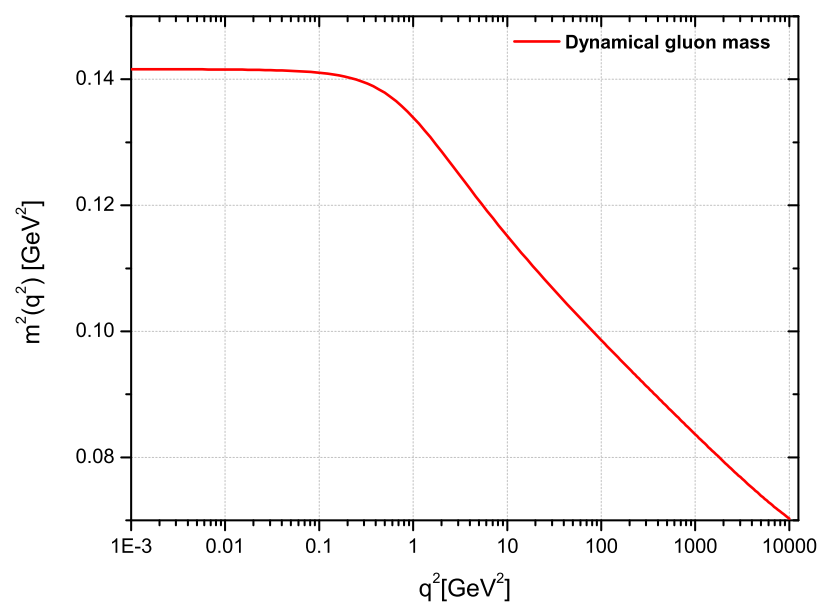

FIG. 19: The square of the dynamical gluon mass, obtained from Eq. (3.23), after plugging into it the $B_{1}^{\prime}(x)$ shown in Fig. 18.

\section{DECOUPLING OF THE MASSLESS EXCITATION: AN EXAMPLE}

In this section we give an explicit example of how the massless excitation decouple from an on-shell amplitude. Specifically, we will show how this is indeed what happens in the case of the four-gluon amplitude. To be sure, a complete proof of the decoupling of the massless excitation from all Yang-Mills amplitudes requires the treatment of kernels with an arbitrary number of incoming gluons, which is beyond our powers at present. However, the example considered here captures the essence of the underlying decoupling mechanism.

The demonstration followed here is similar to that given in [19] for the case of an Abelian model. One starts by considering the complete four-gluon amplitude, [graph $(a)$ in Fig. 20], which consists of three distinct pieces: (i) the amplitude represented by the diagram $(b)$,

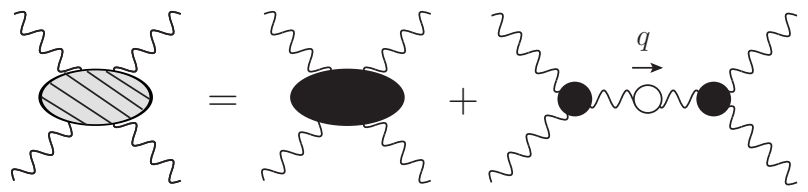

(a)

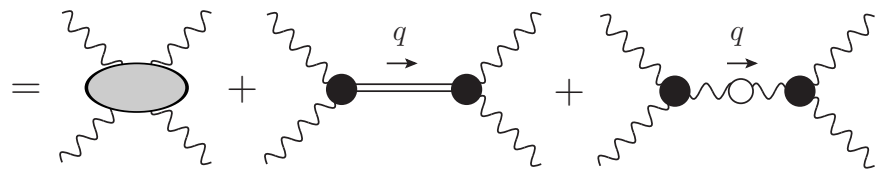

$(b)$

(c)

$(d)$

FIG. 20: The complete four-gluon amplitude and the various terms composing it. 
which is regular as $q^{2} \rightarrow 0$, (ii) the graph $(c)$, which contains the massless excitation, coupled to the external gluons through the proper vertex function $B$, and (iii) the oneparticle reducible term, denoted by $(d)$, which is excluded from the SDE kernel in the usual skeleton expansion. Of course, the above amplitudes are none other than $\left(b_{2}\right),\left(b_{3}\right)$, and (a) in Fig. 3, respectively. Since the amplitude $(b)$ is regular by construction, one must only demonstrate that, as $q^{2} \rightarrow 0$, the divergent part of $(c)$, whose origin is the massless excitation, cancels exactly against an analogous contribution contained in $(d)$, leaving finally a regular result.

We start by considering the term $(d)$. Within the PT-BFM framework that we use, the off-shell gluon (carrying momentum q) is effectively converted into a background gluon; thus, the gluon propagator appearing inside $(d)$ is given by $\widehat{\Delta}(q)$, while the two three-gluon vertices are the $\mathbb{\Gamma}^{\prime}$ defined in Eq. (2.9) . So,

$$
\begin{aligned}
(d) & =-i g^{2} \mathbb{\Gamma}_{\alpha \mu \nu}^{\prime}\left(q, p_{1}, p_{2}\right) P^{\alpha \beta}(q) \widehat{\Delta}(q) \mathbb{\Gamma}_{\beta \rho \sigma}^{\prime}\left(q, p_{3}, p_{4}\right) \\
& =-i g^{2} \mathbb{\Gamma}_{\alpha \mu \nu}^{\prime}\left(q, p_{1}, p_{2}\right) \widehat{\Delta}(q) \mathbb{\Gamma}_{\rho \sigma}^{\alpha}\left(q, p_{3}, p_{4}\right),
\end{aligned}
$$

where the factor $(-i)$ comes from the definition of the gluon propagator, Eq. (2.1). In the second line we have eliminated the longitudinal term $q^{\alpha} q^{\beta} / q^{2}$ inside $P^{\alpha \beta}(q)$ using the "on-shellness" condition

$$
\begin{aligned}
\left.q^{\alpha} \mathbb{\Gamma}_{\alpha \mu \nu}^{\prime}(q, r, p)\right|_{\text {o.s. }} & =\left[\Delta^{-1}\left(p^{2}\right) P_{\mu \nu}(p)-\Delta^{-1}\left(r^{2}\right) P_{\mu \nu}(r)\right]_{\mathrm{o} . \mathrm{s} .} \\
& =0
\end{aligned}
$$

valid for both three-gluon vertices. We emphasize that the full $\mathbb{\Gamma}^{\prime}$ is needed (with the $V$ included) for the on-shellness condition of Eq. (6.2) to be fulfilled. Note also that, if one had chosen a non vanishing gauge-fixing parameter $\xi$ for the gluon propagator (instead of the $\xi=0$ of the Landau gauge), then the condition of Eq. (6.2) is instrumental for the cancellation of the unphysical parameter $\xi$ from the physical amplitude.

Next, it is clear that from the vertex $V$ contained in $\mathbb{\Gamma}^{\prime}$ only the part $U$ survives, [see Eq. (3.3)], because all longitudinal momenta contained in $R$ are annihilated on shell, i.e. when contracted with the appropriate polarization vectors $e_{\mu}(p)$, due to the validity of the relation $p^{\mu} e_{\mu}(p)=0$. Then, we have that (suppressing indices)

$$
\begin{aligned}
\mathbb{\Gamma}^{\prime} \widehat{\Delta} \mathbb{\Gamma}^{\prime} & =(\mathbb{\Gamma}+U) \widehat{\Delta}(\mathbb{\Gamma}+U) \\
& =\mathbb{\Gamma} \widehat{\Delta} \mathbb{\Gamma}+\mathbb{\Gamma}^{\prime} \widehat{\Delta} U+U \widehat{\Delta} \Gamma^{\prime}-U \widehat{\Delta} U
\end{aligned}
$$


Given that the first term in (6.3) is regular, while the second and third term vanish on shell by virtue of (6.2) [which is triggered because $U_{\alpha \mu \nu}$ is proportional to $q_{\alpha}$, see Eq. (3.4)], we are led to the following expression for the pole part of $(d)$

$$
(d)_{\mathrm{pole}}=i g^{2} U_{\alpha \mu \nu} \widehat{\Delta}(q) U_{\rho \sigma}^{\alpha}
$$

Then, using Eqs. (3.7) and (3.9), we obtain

$$
(d)_{\text {pole }}=-\left\{B\left(\frac{i}{q^{2}}\right) B\right\}\left[g^{2} I^{2}(q) \widehat{\Delta}(q)\right] .
$$

Now, in the limit $q^{2} \rightarrow 0$, the quantity in square brackets goes to 1 , precisely by virtue of Eq. (3.41) [remember, $\left.\widehat{\Delta}^{-1}(0)=\widehat{m}^{2}(0)\right]$. Therefore,

$$
\lim _{q^{2} \rightarrow 0}(d)_{\text {pole }}=-\lim _{q^{2} \rightarrow 0}\left\{B\left(\frac{i}{q^{2}}\right) B\right\},
$$

which is precisely the contribution of the term $(c)$ in the same kinematic limit, but with the opposite sign. Therefore, the on-shell four-gluon amplitude is free from poles at $q^{2}=0$, as announced.

Finally, note that, as an alternative, one might opt for eliminating completely any reference to $V$ in the amplitude $(d)$ from the very beginning, namely the first step in Eq. (6.1); this is of course possible, given that some parts of the fully longitudinal vertex $V$ vanish on

shell, while the rest vanishes when contracted with the transverse projector $P_{\alpha \beta}(q)$. In such a case, however, one may not dispose of the longitudinal part of $P_{\alpha \beta}(q)$ any longer, because now the on-shellness condition of Eq. (6.2) is distorted, precisely due to the absence of $V$. It is a straightforward exercise to demonstrate that if one were to take the produced mismatch into account, one would recover exactly the same result found above.

\section{DISCUSSION AND CONCLUSIONS}

The gauge-invariant generation of a gluon mass relies on the existence of massless boundstate excitations, which trigger the Schwinger mechanism. The presence of these excitations in the skeleton expansion of the full three-gluon vertex $\mathbb{\Gamma}_{\alpha \mu \nu}^{\prime}$ induces longitudinally coupled pole structures, giving rise to a purely nonperturbative component, the pole vertex $V_{\alpha \mu \nu}$.

In this article we have studied in detail the dynamical ingredients associated with the vertex $V$; in particular, the poles in $V$ are identified with the propagator of the massless 
scalar excitation, while the tensorial structure is determined by two basic purely nonperturbative quantities: the transition amplitude, denoted by $I_{\alpha}$, which at the diagrammatic level connects the gluon propagator with the massless scalar propagator, and the effective vertex, denoted by $B_{\mu \nu}$, connecting the massless excitation to two outgoing gluons.

The powerful requirement of maintaining the gauge invariance of the theory intact restricts the form of the various form factors composing $B_{\mu \nu}$, and establishes a non-trivial connection between the transition function and the first derivative of the momentum-dependent gluon mass. The insertion of the vertex $V_{\alpha \mu \nu}$ (or, effectively, its surviving component $U_{\alpha \mu \nu}$ ) into the SDE of the gluon propagator (in the Landau gauge) allows one to express, at zero momentum transfer, the gluon mass in terms of the transition function, by means of a rather simple formula. In fact, it turns out that, the relevant dynamical quantity is the derivative of the form factor $B_{1}$, denoted by $B_{1}^{\prime}$.

As we have demonstrated, in the aforementioned kinematic limit, the homogeneous BSE obeyed by the $B_{\mu \nu}$ reduces in a natural way to an analogous integral equation for $B_{1}^{\prime}$. The detailed numerical study of an approximate version of this latter equation reveals the existence of non-trivial solutions for $B_{1}^{\prime}$, which, when inserted into the corresponding formulas, furnish the momentum dependence of the gluon mass. The existence of these solutions adds weight to the hypothesis that the nonperturbative Yang-Mills dynamics lead indeed to the formation of the required massless bound-states.

It is clear that some of the dynamical aspects of this problem merit a further detailed study, due to their relevance in the ongoing scrutiny of the infrared properties of the YangMills Green's functions. Particularly important is to consider the effects of bound-state poles in the SD kernels of not only the three-gluon vertex, as we did in this article, but of all other fundamental vertices of the theory. Such an investigation would involve some or all of the vertices appearing in Eq. (3.14), which would form a coupled system of homogeneous integral equations. Given the recent lattice results on the ghost propagator, especially interesting in this context is the dynamical information that one might be able to obtain about the quantity $B$, corresponding to the wave-function of the ghost-ghost channel [(vertex $a_{1}$ in Fig. (44)]. Specifically, while the ghost dressing function $F$ is found to be finite in the infrared, a fact that can be explained by the presence of the gluon mass that saturates the corresponding perturbative logarithm, there is no dynamical mass associated with the ghost field. One would expect, therefore, that the solution of the corresponding system should give rise to a 
non-vanishing $B_{1}^{\prime}$, as before, but to a vanishing ghost-ghost wave function $B$.

As has been explained in detail in Section II, the incorporation the massless excitations modify the three-gluon vertex $\mathbb{\Gamma}$, giving rise to the new vertex $\mathbb{\Gamma}^{\prime}$, defined in Eq. (2.9) . It would certainly be particularly interesting to compare the characteristic features of $\mathbb{\Gamma}^{\prime}$ with results obtained on the lattice for the three-gluon vertex [43]. In particular, one might in principle be able to relate the presence of the massless poles to possible divergences of some of the form factors, in the appropriate kinematic limit. To that end, one must first determine the closed expression for the entire vertex $V$ from Eq. (2.8) and the WI and STIs it satisfies. Then the answer should be written in a standard basis, such that of Ball and Chiu [44, 45], and the final result projected on the particular kinematic configurations usually employed in the lattice calculations. We hope to be able to carry out this project in the near future.

\section{Acknowledgments}

The research of D.I. and J.P. is supported by the European FEDER and Spanish MICINN under grant FPA2008-02878, and that of V.M. under grant FPA2010-21750-C02-01. The work of A.C.A. is supported by the Brazilian Funding Agency CNPq under the grant 305850/2009-1 and project 474826/2010-4.

[1] A. Cucchieri and T. Mendes, PoS LAT2007, 297 (2007).

[2] A. Cucchieri and T. Mendes, Phys. Rev. Lett. 100, 241601 (2008).

[3] A. Cucchieri and T. Mendes, Phys. Rev. D 81, 016005 (2010).

[4] A. Cucchieri and T. Mendes, PoS LATTICE2010, 280 (2010).

[5] A. Cucchieri and T. Mendes, AIP Conf. Proc. 1343, 185 (2011).

[6] I. L. Bogolubsky, E. M. Ilgenfritz, M. Muller-Preussker and A. Sternbeck, PoS LATTICE, 290 (2007).

[7] P. O. Bowman et al., Phys. Rev. D 76, 094505 (2007).

[8] I. L. Bogolubsky, E. M. Ilgenfritz, M. Muller-Preussker and A. Sternbeck, Phys. Lett. B 676, 69 (2009).

[9] O. Oliveira and P. J. Silva, PoS LAT2009, 226 (2009). 
[10] J. M. Cornwall, Phys. Rev. D 26, 1453 (1982).

[11] A. C. Aguilar, A. A. Natale, P. S. Rodrigues da Silva, Phys. Rev. Lett. 90, 152001 (2003).

[12] A. C. Aguilar and J. Papavassiliou, JHEP 0612, 012 (2006).

[13] A. C. Aguilar, D. Binosi and J. Papavassiliou, Phys. Rev. D 78, 025010 (2008).

[14] A. C. Aguilar, J. Papavassiliou, Phys. Rev. D81, 034003 (2010).

[15] A. C. Aguilar, D. Binosi and J. Papavassiliou, arXiv:1107.3968 [hep-ph].

[16] J. S. Schwinger, Phys. Rev. 125, 397 (1962).

[17] J. S. Schwinger, Phys. Rev. 128, 2425 (1962).

[18] R. Jackiw and K. Johnson, Phys. Rev. D 8, 2386 (1973).

[19] R. Jackiw, In *Erice 1973, Proceedings, Laws Of Hadronic Matter*, New York 1975, 225-251 and M I T Cambridge - COO-3069-190 (73,REC.AUG 74) 23p.

[20] J. M. Cornwall and R. E. Norton, Phys. Rev. D 83338 (1973).

[21] E. Eichten and F. Feinberg, Phys. Rev. D 10, 3254 (1974).

[22] E. C. Poggio, E. Tomboulis and S. H. Tye, Phys. Rev. D 11, 2839 (1975)).

[23] J. Rodriguez-Quintero, PoS LC2010, 023 (2010); AIP Conf. Proc. 1354, 118 (2011).

[24] Ph. Boucaud, J. P. Leroy, A. L. Yaouanc, J. Micheli, O. Pene and J. Rodriguez-Quintero, JHEP 0806 (2008) 012.

[25] D. Dudal, J. A. Gracey, S. P. Sorella, N. Vandersickel and H. Verschelde, Phys. Rev. D 78, 065047 (2008).

[26] C. S. Fischer, A. Maas, J. M. Pawlowski, Annals Phys. 324, 2408-2437 (2009).

[27] K. -I. Kondo, Prog. Theor. Phys. 122, 1455-1475 (2010); arXiv:0909.4866 [hep-th]].

[28] O. Oliveira and P. Bicudo, J. Phys. G 38, 045003 (2011).

[29] M. R. Pennington, D. J. Wilson, arXiv:1109.2117 [hep-ph]].

[30] J. M. Cornwall and J. Papavassiliou, Phys. Rev. D 40, 3474 (1989).

[31] A. Pilaftsis, Nucl. Phys. B 487, 467 (1997).

[32] D. Binosi, J. Papavassiliou, Phys. Rev. D66, 111901 (2002).

[33] D. Binosi, J. Papavassiliou, J. Phys. G G30, 203 (2004).

[34] D. Binosi and J. Papavassiliou, Phys. Rept. 479, 1-152 (2009).

[35] L. F. Abbott, Nucl. Phys. B 185, 189 (1981), and references therein.

[36] D. Binosi, J. Papavassiliou, JHEP 0811, 063 (2008).

[37] P. A. Grassi, T. Hurth and M. Steinhauser, Annals Phys. 288, 197 (2001). 
[38] D. Binosi and J. Papavassiliou, Phys. Rev. D 66, 025024 (2002).

[39] T. Kugo and I. Ojima, Prog. Theor. Phys. Suppl. 66, 1 (1979).

[40] P. A. Grassi, T. Hurth and A. Quadri, Phys. Rev. D 70, 105014 (2004).

[41] K. I. Kondo, arXiv:0904.4897 [hep-th].

[42] A. C. Aguilar, D. Binosi, J. Papavassiliou, J. Rodriguez-Quintero, Phys. Rev. D80, 085018 (2009).

[43] A. Cucchieri, A. Maas, T. Mendes, Phys. Rev. D77, 094510 (2008).

[44] J. S. Ball and T. W. Chiu, Phys. Rev. D 22, 2550 (1980) [Erratum-ibid. D 23, 3085 (1981)].

[45] D. Binosi and J. Papavassiliou, JHEP 1103, 121 (2011). 